\title{
Application of light therapy in older adults with cognitive impairment: A systematic review
}

\author{
Nuria Cibeira ${ }^{1}$, Ana Maseda ${ }^{1}$, Laura Lorenzo-López ${ }^{1}$, José L. Rodríguez- \\ Villamil $^{1}$, Rocío López-López ${ }^{1}$, José C. Millán-Calenti ${ }^{1}$ \\ ${ }^{1}$ Universidade da Coruña, Gerontology and Geriatrics Research Group, Instituto de Investigación \\ Biomédica de A Coruña (INIBIC), Complexo Hospitalario Universitario de A Coruña (CHUAC), SERGAS, \\ 15071 A Coruña, Spain.
}

\begin{abstract}
This systematic review aims to assess the efficacy of light therapy on behavioural and psychological symptoms of dementia (BPSD), cognition, functional status, and quality of life in older adults with cognitive impairment; and secondarily, to identify the optimal characteristics of light therapy to establish an adequate protocol for its clinical application. We searched Web of Science and Medline databases through December 2019, resulting in 36 included articles: 3 evaluated the effects on BPSD, 25 on sleep, 12 on agitation, 10 on mood, 4 on neuropsychiatric symptoms, 4 on cognition, 2 on quality of life and 2 on functional status. Literature has shown potential evidence for positive effects of light therapy on managing sleep, behavioural and mood disturbances in people with cognitive impairment, but a limited effect on cognition, quality of life and functional status. This review provides guidelines for intervention protocols with light therapy in older people with cognitive impairment.
\end{abstract}

\section{Keywords}

Light therapy; Dementia; Cognitive impairment; Older adults 


\section{Introduction}

The number of people affected by dementia worldwide is 50 million and will continue to increase dramatically in the next decades, representing one of the most important public health problems.1 It is the main reason for the functional dependence and institutionalization of older adults, with a high economic impact on society. ${ }^{2}$

Dementia affects cognitive function and is usually accompanied by the presence of behavioural and psychological symptoms of dementia, BPSD, ${ }^{3}$ which comprise four factors: a psychosis factor- irritability, agitation, hallucinations, and anxiety; a psychomotor factor- aberrant motor behaviour and delusions; a mood liability factor- disinhibition, elation and depression; and an instinctual factor- appetite disorders, sleep disorders and apathy. ${ }^{4}$ The most prevalent BPSD are apathy, depression, irritability, agitation, and anxiety, while the less prevalent ones are euphoria, hallucinations, and disinhibition. ${ }^{5}$ It is important to note that the prevalence of BPSD may vary in each subtype of dementia (see a review ${ }^{5}$ ), patients with vascular dementia have reported a higher prevalence and severity of depression and anxiety, or patients with dementia with Lewy bodies a higher prevalence of delusions and hallucinations, in comparison to Alzheimer's Disease, with a higher prevalence of aberrant motor behaviour.

BPSD are the most distressing symptoms of dementia for caregivers and contribute to institutionalization, ${ }^{6}$ with agitation being one of the most relevant symptoms, affecting both formal and informal caregivers. ${ }^{7}$ Sleep disturbances also represent an important clinical problem in dementia and are the main contributor to caregiver distress. ${ }^{8}$

Accurate BPSD characterization and intervention are key factors in the treatment of dementia, including a combination of pharmacological and non-pharmacological interventions. ${ }^{5}$ BPSD management is a very difficult task for formal and informal caregivers due to possible adverse events or a lack of research supporting the use of different interventions. ${ }^{3}$ Pharmacological and non-pharmacological interventions have been proposed for the treatment of both BPSD and cognitive impairment, and although the former (mainly antipsychotics) have demonstrated a slightly larger effect size, non-pharmacological strategies should be initially recommended to manage dementia (see a review ${ }^{9}$ ). In fact, the American Geriatrics Society Beers Criteria Update Expert Panel ${ }^{10}$ recommends, "antipsychotics must be avoided for BPSD unless nonpharmacological options have failed or are not possible and the older adult is threatening substantial harm to self or others". Therefore, alternative interventions are being used as a nonpharmacological approach to symptoms associated with dementia due to the lack of adverse events (see reviews ${ }^{9,11}$ such as individualized music, ${ }^{12}$ multisensory stimulation, ${ }^{13}$ aromatherapy, ${ }^{14}$ reminiscence therapy, ${ }^{15}$ and light therapy ${ }^{16,17}$ ).

Light therapy offers a promising non-pharmacological alternative in people with dementia to treat the physiological changes associated with alterations of circadian rhythm. ${ }^{18}$ Older adults with dementia have a decreased general sensory input, low sensitivity to the light effect on the suprachiasmatic nucleus (SCN) and less exposure to bright environmental light. ${ }^{18}$ Light therapy might reverse the degenerative changes in the SCN, which are the biological basis for circadian (rest-activity and sleep-wake cycles) disturbances in people with dementia. ${ }^{18}$ Most institutional settings maintain consistent low light levels, which may not be sufficient to enable the circadian clock to entrain to the 24-hour day. ${ }^{19}$ There is evidence that effective light doses would stimulate the circadian cycles, affecting sleep efficiency (see a review20), depression, ${ }^{21}$ functional status ${ }^{22}$ or behavioural problems ${ }^{23,24}$ in older adults with dementia. Light therapy involves exposure under controlled conditions to certain levels of light. There are different forms of administering light therapy, using outdoor sunlight or artificial indoor light sources. Different light sources or devices can provide it: light boxes placed approximately one meter away from the subjects at a height within their visual fields; a light visor worn on their heads; or a more acceptable 'naturalistic' light therapy, known as a dawn-dusk simulation that mimics outdoor twilight transitions. ${ }^{25}$ In all cases, light needs to enter the user's eyes to be effective, however it is not necessary to stare at the light 
source, even looking at lights is not recommended with some devices. ${ }^{26}$ People may do other activities while receiving the light stimulation in their visual field, such as eating, watching television or reading, being easily implementable either in institutions or in dwellings. Additionally, there is wide methodological heterogeneity with regard to the light intensity, frequency, interval, time of the day, and length of the intervention among the studies. ${ }^{18}$

Importantly, limited research has been conducted to examine the efficacy of light therapy in managing BPSD in older adults with cognitive impairment. In fact, in the most recent review ${ }^{17}$ examining light therapy, the authors did only involve participants with Alzheimer's disease. Therefore, this systematic review differs from others in providing an overview of the existing literature to date that examine the effect of light on BPSD, cognition, functional status, and quality of life in participants with all degrees of cognitive impairment. Moreover, this review pursues to provide a more practical point of view in order to facilitate the implementation of light therapy protocols in daily clinical practice.

The aim of the present systematic review is to assess studies exploring the efficacy of light therapy as a non-pharmacological approach to manage BPSD, as well as its effectiveness in cognition, functional status, and quality of life in people with cognitive impairment (not necessarily with a diagnosis of dementia). Additionally, we aim to identify the light therapy conditions with the highest efficacy for older adults with cognitive impairment in order to facilitate the establishment of an adequate protocol for the clinical application of light therapy in this population.

\section{Materials and methods}

The Preferred Reporting Items for Systematic Reviews and Meta-analysis (PRISMA) guidelines were used for reporting of this systematic review. ${ }^{27}$

\section{Search strategy}

We identified articles that assessed the effect of light therapy for older adults with cognitive impairment by searching the Web of Science and Medline databases. The search included articles published until December 2019. Search terms included ("light therapy" or "light treatment" or "light exposure" or "bright light" or "effect of light"), and ("elder*" or "old*” or "dement*" or "Alzheimer" or "nursing home" or "day care"). Combinations of these terms were used for the title search (see search strategy in Appendix A published as supplementary material online). All possible articles were merged into a single file, and duplicate records were removed after they were checked manually. Two of the investigators independently evaluated the appropriateness of inclusion, and discrepancies were discussed and solved to reach a consensus. If disagreements arose, a third investigator was included in the discussion to reach the final consensus.

\section{Study selection}

The following inclusion criteria were established to select articles for this systematic review. Regarding participants: individuals with a mean age of 65 years or older with cognitive impairment (mild cognitive impairment and mild, moderate or severe dementia). In terms of the intervention: any type of light therapies, with specific information about duration and frequency. As regards the outcome measures: the effect on BPSD (especially with regard to sleep and agitation), cognitive status, functional status or quality of life as the primary outcome. Finally, 
with regard to language: only full-text articles written in English or Spanish were eligible. As exclusion criteria: reviews, letters, editorials, conference abstracts or papers, corrections or book chapters were excluded.

\section{Quality assessment and data extraction}

This systematic review adheres to the guidelines detailed on the PRISMA protocol. ${ }^{27}$ The quality of the selected papers was assessed by two independent researchers, using two Critical Appraisal Tools provided by the Joanna Briggs Institute (JBI) ${ }^{28}$ with a third investigator in case of any disagreements, likewise done for the included studies. JBI Critical Appraisal Checklist for Randomized Controlled Trials, with 13 items, was used to assess 18 articles. The other 18 articles were evaluated by JBI Critical Appraisal Checklist for Quasi-Experimental Studies (nonrandomized experimental studies), with 9 items. At the end of the quality assessment of each article, an overall score of appraisal is determined based on the criteria number ranked as "Yes".

Data of each article included were collected according to the following characteristics: authors and year of publication, study design and intervention setting (gerontological complex, community-dwelling, hospital...), sample characteristics (age and sex), cognitive status, intervention characteristics (intensity, frequency, and duration), assessed outcomes, measurement tools, and main findings.

This systematic review did not require ethics approval from an ethics committee.

Due to the heterogeneity of the results, the measures and the design, a meta-analysis was not appropriate in this case.

\section{Results}

Fig. 1 includes the PRISMA flow chart of the study selection process. The search of Web of Science produced 208 results and of Medline produced 152 results. Moreover, 4 additional records were identified through the bibliography of some of the analysed articles. Of the 246 studies identified after removing duplicates, 101 remained as potentially relevant and were analysed in terms of their eligibility. Of these studies, 35 were excluded for the setting, 5 for not being focused on BPSD, cognitive status or quality of life, 9 for insufficient reporting of light therapy and 16 for not being original research (see Appendix B published as supplementary material online). Finally, 36 articles met the inclusion criteria for this review. 


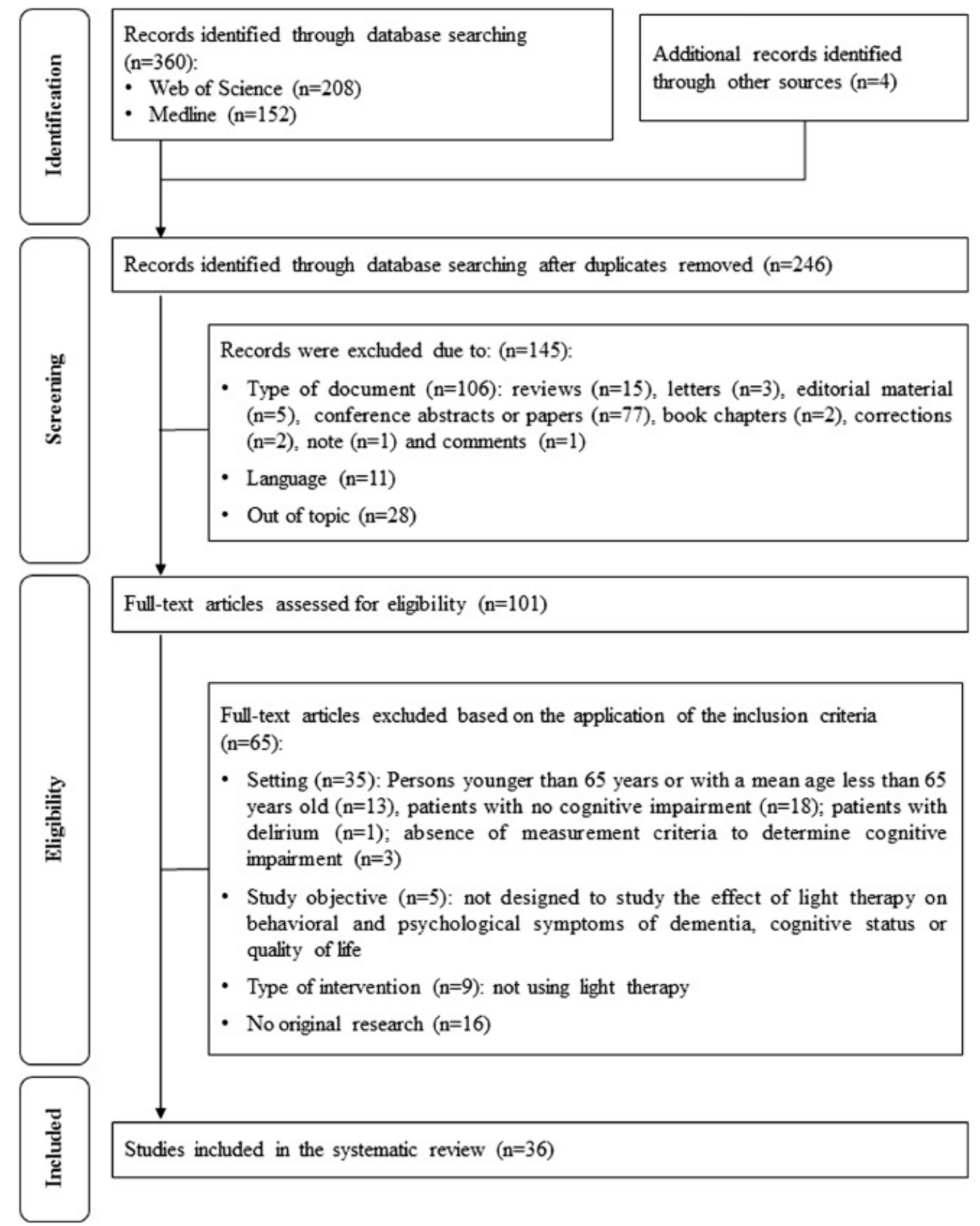

Fig 1. PRISMA Flow Diagram of literature search and selection process of the current systematic review.

Table 1 summarizes the characteristics of the included studies. As can be seen in the table, multiple articles, based on the same study sample, but describing different outcomes, were included in this systematic review. 
Table 1. Characteristics of the included studies in the systematic review.

\begin{tabular}{|c|c|c|c|c|c|c|c|}
\hline Author (year) & $\begin{array}{l}\text { Study design/ } \\
\text { Setting }\end{array}$ & Sample & Cognitive status & Intervention characteristics & $\begin{array}{l}\text { Outcome } \\
\text { measured }\end{array}$ & $\begin{array}{l}\text { Measurement } \\
\text { tools }\end{array}$ & Main findings \\
\hline $\begin{array}{l}\text { Ancoli-Israel } \\
(2002)^{54}\end{array}$ & $\begin{array}{l}\text { RCT } \\
\text { Nursing home }\end{array}$ & $\begin{array}{l}n=77 \text { (58 women) } \\
\text { Mean age }=85 . \\
7 \pm 7.3\end{array}$ & $\begin{array}{l}\text { Mean MMSE }=12.8 \pm 8.8 \\
(\text { range }=0-30)\end{array}$ & $\begin{array}{l}\text { Four conditions: EBL, MBL, } \\
\text { daytime sleep restriction, } \\
\text { DRL. } \\
\text { Procedure: BL ( } 2500 \text { lux), } \\
\text { DRL (<50 lux); } 18 \text { d; } 2 \text { h/d }\end{array}$ & $\begin{array}{l}\text { Sleep, } \\
\text { Circadian } \\
\text { rhythm }\end{array}$ & Actigraphy & $\begin{array}{l}\text { No improvements in night-time sleep or daytime } \\
\text { alertness. Morning bright light: a delayed peak of } \\
\text { the activity rhythm, increased mean activity level } \\
\text { and improved activity rhythmicity }\end{array}$ \\
\hline $\begin{array}{l}\text { Ancoli-Israel } \\
(2003 a)^{55 *}\end{array}$ & $\begin{array}{l}\text { RCT } \\
\text { Nursing home }\end{array}$ & $\begin{array}{l}n=92(63 \text { women }) \\
\text { Mean age }=82 . \\
3 \pm 7.6\end{array}$ & $\begin{array}{l}\text { Probable or possible AD, } \\
\text { mean MMSE }=5.7 \pm 5.6 \\
\text { (range }=0-22)\end{array}$ & $\begin{array}{l}\text { Three conditions: MBL, } \\
\text { morning DRL, EBL. } \\
\text { Procedure: BL ( } 2500 \text { lux), } \\
\text { DRL ( }<300 \text { lux); } 10 \mathrm{~d} ; 2 \text { h/d }\end{array}$ & Agitation & CMAI, ABRS & $\begin{array}{l}\text { CMAI: significant improvement in physical, } \\
\text { verbal and overall agitation caregiver ratings of } \\
\text { three treatment groups; ABRS: no significant } \\
\text { changes }\end{array}$ \\
\hline $\begin{array}{l}\text { Ancoli-Israel } \\
(2003 b)^{56 *}\end{array}$ & $\begin{array}{l}\text { RCT } \\
\text { Nursing home }\end{array}$ & $\begin{array}{l}n=92(63 \text { women }) \\
\text { Mean age }=82 . \\
3 \pm 7.6\end{array}$ & $\begin{array}{l}\text { Probable or possible AD, } \\
\text { mean MMSE }=5.7 \pm 5.6 \\
\text { (range }=0-22)\end{array}$ & $\begin{array}{l}\text { Three conditions: MBL, } \\
\text { morning DRL, EBL. } \\
\text { Procedure: BL ( } 2500 \text { lux), } \\
\text { DRL ( }<300 \text { lux); } 10 \text { d; } 2 \text { h/d }\end{array}$ & Sleep & Actigraphy & $\begin{array}{l}\text { Increased MBL or EBL consolidates night-time } \\
\text { sleep by lengthening the maximum sleep bouts } \\
\text { during the night. Time asleep did not vary as there } \\
\text { were longer but fewer sleep bouts }\end{array}$ \\
\hline $\begin{array}{l}\text { Barrick } \\
(2010)^{31} \dagger\end{array}$ & $\begin{array}{l}\text { Cluster-unit } \\
\text { crossover design } \\
\text { Psychiatric } \\
\text { hospital and } \\
\text { nursing home }\end{array}$ & $\begin{array}{l}n=66 \text { ( } 31 \text { women }) \\
\text { Mean age }=79\end{array}$ & $\begin{array}{l}\text { Dementia, MDS- } \\
\text { COGS/MMSE: Mild: } n=3 \text {; } \\
\text { Moderate: } n=18 \text {; } \\
\text { Severe: } n=31 \text {; Very } \\
\text { severe: } n=14\end{array}$ & $\begin{array}{l}\text { Four conditions: MBL, EBL; } \\
\text { all day BL, STL. } \\
\text { Procedure: BL ( } 2000- \\
3000 \text { lux), STL ( } 500- \\
600 \text { lux); 3 weeks; MBL EBL: } \\
4 \text { h/d, all day BL: } 13 \text { h/d }\end{array}$ & Agitation & CMAI & $\begin{array}{l}\text { BL was not effective in reducing agitation under } \\
\text { any intervention condition, even exacerbating it }\end{array}$ \\
\hline $\begin{array}{l}\text { Burns } \\
(2009)^{51}\end{array}$ & $\begin{array}{l}\text { RCT } \\
\text { Nursing home }\end{array}$ & $\begin{array}{l}n=48 \text { (32 women) } \\
\text { Mean } \\
\text { age }=83,5 \pm 1,6\end{array}$ & $\begin{array}{l}\text { Dementia diagnosis, mean } \\
\text { MMSE: } \text { STL group }=5 . \\
1 \pm 5.6 \text {; } \text { BL group }=6 . \\
9 \pm 5.3\end{array}$ & $\begin{array}{l}\text { Two conditions: MBL and } \\
\text { STL. } \\
\text { Procedure: MBL (10,000 lux), } \\
\text { STL (100 lux); } 2 \text { weeks; } 2 \text { h/d }\end{array}$ & $\begin{array}{l}\text { Agitation, } \\
\text { Cognition, } \\
\text { Mood, Sleep }\end{array}$ & $\begin{array}{l}\text { CMAI, MMSE, } \\
\text { CRBRS, CSDD, } \\
\text { MOUSEPAD, } \\
\text { Sleep charts, } \\
\text { Actigraphy }\end{array}$ & $\begin{array}{l}\text { Limited evidence of a reduction in agitation. Sleep } \\
\text { and diurnal rhythm are improved, suggesting } \\
\text { greater efficacy during the winter season }\end{array}$ \\
\hline 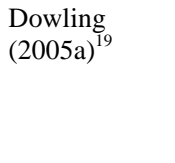 & $\begin{array}{l}\text { RCT } \\
\text { Nursing home }\end{array}$ & $\begin{array}{l}n=46 \text { ( } 36 \text { women }) \\
\text { Mean age }=84 \\
0 \pm 10.0\end{array}$ & $\begin{array}{l}\text { AD diagnosis, mean } \\
\mathrm{MMSE}=6.7 \pm 6.8\end{array}$ & $\begin{array}{l}\text { Two conditions: MBL and } \\
\text { STL. } \\
\text { Procedure: MBL ( } \geq 2500 \text { lux), } \\
\text { STL (150-200 lux); } 10 \text { weeks; } \\
1 \text { h/d }\end{array}$ & Sleep & Actigraphy & $\begin{array}{l}\text { Significant improvement in rest-activity rhythms } \\
\text { only in subjects with the most impaired rest- } \\
\text { activity rhythms }\end{array}$ \\
\hline
\end{tabular}




\begin{tabular}{|c|c|c|c|c|c|c|c|}
\hline Author (year) & $\begin{array}{l}\text { Study design/ } \\
\text { Setting }\end{array}$ & Sample & Cognitive status & Intervention characteristics & $\begin{array}{l}\text { Outcome } \\
\text { measured }\end{array}$ & $\begin{array}{l}\text { Measurement } \\
\text { tools }\end{array}$ & Main findings \\
\hline $\begin{array}{l}\text { Dowling } \\
\text { (2005b) })^{57} \ddagger\end{array}$ & $\begin{array}{l}\text { RCT } \\
\text { Nursing home }\end{array}$ & $\begin{array}{l}n=70(57 \text { women }) \\
\text { Mean age }=84 . \\
0 \pm 10.0\end{array}$ & $\begin{array}{l}\text { AD diagnosis, mean } \\
\mathrm{MMSE}=7.0 \pm 7.0\end{array}$ & $\begin{array}{l}\text { Three conditions: MBL, EBL, } \\
\text { STL. } \\
\text { Procedure: BL ( } \geq 2500 \text { lux), } \\
\text { STL (150-200 lux); } 10 \text { weeks; } \\
1 \text { h/d }\end{array}$ & Sleep & Actigraphy & $\begin{array}{l}\text { No significant differences in night-time sleep or } \\
\text { day-time wakefulness. MBL implies a } \\
\text { significantly more stable rest-activity rhythm }\end{array}$ \\
\hline $\begin{array}{l}\text { Dowling } \\
(2007)^{58} \ddagger\end{array}$ & $\begin{array}{l}\text { RCT } \\
\text { Nursing home }\end{array}$ & $\begin{array}{l}n=70(57 \text { women }) \\
\text { Mean age }=84 . \\
0 \pm 10.0\end{array}$ & $\begin{array}{l}\text { AD diagnosis, mean } \\
\text { MMSE }=7.0 \pm 7.0\end{array}$ & $\begin{array}{l}\text { Three conditions: MBL, EBL, } \\
\text { STL. } \\
\text { Procedure: BL ( } \geq 2500 \text { lux), } \\
\text { STL (150-200 lux); } 10 \text { weeks; } \\
1 \text { h/d }\end{array}$ & NPS & NPI - NH & $\begin{array}{l}\text { Significant differences in depression/dysphoria, } \\
\text { aberrant motor behaviour, and eating disorders, } \\
\text { but with a small change, not indicative of a } \\
\text { clinical effect }\end{array}$ \\
\hline $\begin{array}{l}\text { Dowling } \\
(2008)^{59}\end{array}$ & $\begin{array}{l}\text { RCT } \\
\text { Nursing home }\end{array}$ & $\begin{array}{l}n=50 \text { ( } 43 \text { women }) \\
\text { Mean age }=86 . \\
0 \pm 8.0\end{array}$ & $\begin{array}{l}\text { AD diagnosis, mean } \\
\text { MMSE }=9.3 \pm 7.9\end{array}$ & $\begin{array}{l}\text { Three conditions: } \\
\text { MBL + melatonin, } \\
\text { MBL + placebo, STL. } \\
\text { Procedure: MBL ( } \geq 2500 \text { lux), } \\
\text { STL (150-200 lux); } 10 \text { weeks; } \\
1 \text { h/d }\end{array}$ & Sleep & Actigraphy & $\begin{array}{l}\text { MBL plus melatonin significantly improves day- } \\
\text { time somnolence, reduces the duration of day-time } \\
\text { sleep, increases day-time activity, and improves } \\
\text { the day: night sleep ratio }\end{array}$ \\
\hline $\begin{array}{l}\text { Fetveit } \\
(2003){ }^{46} \S\end{array}$ & $\begin{array}{l}\text { Repeated } \\
\text { measures design } \\
\text { Nursing home }\end{array}$ & $\begin{array}{l}n=11(10 \text { women }) \\
\text { Mean age }=86 . \\
1 \pm 8.9\end{array}$ & $\begin{array}{l}\text { Moderate to severe } \\
\text { dementia, mean } \\
\text { MMSE }=11.7 \pm 4.2 \text {; Mean } \\
\text { CDR }=2.5 \pm 0.5\end{array}$ & $\begin{array}{l}\text { One condition: MBL. } \\
\text { Procedure: 6000-8000 lux; } 2 \\
\text { weeks; } 2 \text { h/d }\end{array}$ & Sleep & $\begin{array}{l}\text { Actigraphy, Own } \\
\text { scale of sleep- } \\
\text { wake } \\
\text { disturbances }\end{array}$ & $\begin{array}{l}\text { Significant improvements in nocturnal sleep, sleep } \\
\text { maintenance and sleep efficiency }\end{array}$ \\
\hline $\begin{array}{l}\text { Fetveit } \\
(2004){ }^{47} \S\end{array}$ & $\begin{array}{l}\text { Repeated } \\
\text { measures design } \\
\text { Nursing home }\end{array}$ & $\begin{array}{l}n=11(10 \text { women }) \\
\text { Mean age }=86 . \\
1 \pm 8.9\end{array}$ & $\begin{array}{l}\text { Moderate to severe } \\
\text { dementia, mean } \\
\text { MMSE }=11.7 \pm 4.2 \text {; Mean } \\
\text { CDR }=2.5 \pm 0.5\end{array}$ & $\begin{array}{l}\text { One condition: MBL. } \\
\text { Procedure: 6000-8000 lux; } 2 \\
\text { weeks; } 2 \text { h/d }\end{array}$ & Sleep & Actigraphy & $\begin{array}{l}\text { Significant positive effects on sleep disturbances } \\
\text { (sleep onset latency, nocturnal wake time and } \\
\text { efficiency), even at the } 12 \text {-week follow-up }\end{array}$ \\
\hline $\begin{array}{l}\text { Fetveit } \\
(2005){ }^{48} \S\end{array}$ & $\begin{array}{l}\text { Repeated } \\
\text { measures design } \\
\text { Nursing home }\end{array}$ & $\begin{array}{l}n=11(10 \text { women }) \\
\text { Mean age }=86 . \\
1 \pm 8.9\end{array}$ & $\begin{array}{l}\text { Moderate to severe } \\
\text { dementia, mean } \\
\text { MMSE }=11.7 \pm 4.2 \text {; Mean } \\
\text { CDR }=2.5 \pm 0.5\end{array}$ & $\begin{array}{l}\text { One condition: MBL. } \\
\text { Procedure: } 6000-8000 \text { lux; } 2 \\
\text { weeks; } 2 \text { h/d }\end{array}$ & Sleep & $\begin{array}{l}\text { Actigraphy, Own } \\
\text { scale of sleep- } \\
\text { wake } \\
\text { disturbances }\end{array}$ & $\begin{array}{l}\text { Significant positive effect on daytime } \\
\text { wakefulness, decreasing daytime sleep }\end{array}$ \\
\hline $\begin{array}{l}\text { Figueiro } \\
(2014)^{64}\end{array}$ & $\begin{array}{l}\text { Repeated } \\
\text { measures } \\
\text { Nursing home }\end{array}$ & $\begin{array}{l}n=14 \text { (9 women }) \\
\text { Mean age }=86 \\
9 \pm 4.4\end{array}$ & $\begin{array}{l}\text { Mild-moderate dementia, } \\
\text { mean BIMS }=7.7 \pm 2.3\end{array}$ & $\begin{array}{l}\text { One condition: Bluish-white } \\
\text { light. } \\
\text { Procedure: } 324 \pm 190 \text { lux; } 4 \\
\text { weeks; } 8-10 \text { h/d }\end{array}$ & $\begin{array}{l}\text { Sleep, } \\
\text { Circadian } \\
\text { rhythm, } \\
\text { Mood, } \\
\text { Agitation, } \\
\text { ADL }\end{array}$ & $\begin{array}{l}\text { Daysimeter, } \\
\text { PSQI, MSD- } \\
\text { ADL, CSDD, } \\
\text { CMAI }\end{array}$ & $\begin{array}{l}\text { Significant increase in total sleep time and sleep } \\
\text { efficiency. Significant improvement in sleep, } \\
\text { depression and agitation scores from the } \\
\text { standardized questionnaires applied }\end{array}$ \\
\hline
\end{tabular}




\begin{tabular}{|c|c|c|c|c|c|c|c|}
\hline Author (year) & $\begin{array}{l}\text { Study design/ } \\
\text { Setting }\end{array}$ & Sample & Cognitive status & Intervention characteristics & $\begin{array}{l}\text { Outcome } \\
\text { measured }\end{array}$ & $\begin{array}{l}\text { Measurement } \\
\text { tools }\end{array}$ & Main findings \\
\hline $\begin{array}{l}\text { Figueiro } \\
(2015)^{32}\end{array}$ & $\begin{array}{l}\text { Repeated } \\
\text { measures } \\
\text { Community- } \\
\text { dwelling }\end{array}$ & $\begin{array}{l}=35 \text { ( } 9 \text { women }) \text { and } \\
34 \text { caregivers }(27 \\
\text { women) } \\
\text { Mean age }=80 . \\
8 \pm 7.9\end{array}$ & $\begin{array}{l}\text { Mild-moderate dementia, } \\
\text { MMSE = range } 12-24 ; \\
\text { CDR = range } 1-2\end{array}$ & $\begin{array}{l}\text { One condition: Bluish-white } \\
\text { light. } \\
\text { Procedure: } 350-400 \text { lux; } 4 \\
\text { weeks; } 8-10 \text { h/d }\end{array}$ & $\begin{array}{l}\text { Circadian } \\
\text { rhythm, } \\
\text { Sleep, Mood }\end{array}$ & $\begin{array}{l}\text { Daysimeter, } \\
\text { Actigraphy, } \\
\text { Sleep diary, } \\
\text { PSQI, CSDD, } \\
\text { GDS-SF }\end{array}$ & $\begin{array}{l}\text { Significant increase in circadian entrainment in } \\
\text { both, participants with dementia and their } \\
\text { caregivers. Participants with dementia also } \\
\text { showed an improvement in sleep efficiency and } \\
\text { depressive symptoms }\end{array}$ \\
\hline $\begin{array}{l}\text { Fontana } \\
\text { Gasio } \\
(2003)^{49}\end{array}$ & $\begin{array}{l}\text { RCT } \\
\text { Nursing home }\end{array}$ & $\begin{array}{l}n=13 \text { ( } 12 \text { women }) \\
\text { Mean } \\
\text { age }=84,9 \pm 4,8\end{array}$ & $\begin{array}{l}\text { Diagnosis of dementia, } \\
\text { mean MMSE: DDS } \\
\text { group = 13. } 8 \pm 5.9 ; \\
\text { placebo group }=14.3 \pm 4.1\end{array}$ & $\begin{array}{l}\text { Two conditions: DDS and } \\
\text { placebo (DRL). } \\
\text { Procedure: DDS (max. } \\
400 \text { lux), DRL (<5 lux); } 3 \\
\text { weeks; per day: dawn time } \\
\text { and dusk time }\end{array}$ & $\begin{array}{l}\text { Circadian } \\
\text { rhythm, } \\
\text { Sleep, } \\
\text { Cognition, } \\
\text { NPS }\end{array}$ & $\begin{array}{l}\text { Actigraphy, } \\
\text { MMSE, } \\
\text { CERAD, NPI- } \\
\text { NH, GDS-SF }\end{array}$ & $\begin{array}{l}\text { DDS group presented shortened sleep latency, } \\
\text { longer sleep duration, more nocturnal immobility } \\
\text { and less nocturnal activity than the DRL group. } \\
\text { No significant effects of either condition in the } \\
\text { neuropsychological evaluations }\end{array}$ \\
\hline $\begin{array}{l}\text { Friedman } \\
(2012)^{33}\end{array}$ & $\begin{array}{l}\text { Parallel group } \\
\text { design } \\
\text { Community- } \\
\text { dwelling }\end{array}$ & $\begin{array}{l}n=54(23 \text { women }) \\
\text { Mean age }=77 \\
9 \pm 8.1\end{array}$ & $\begin{array}{l}\text { Mild cognitive impairment } \\
\text { to dementia, mean } \\
\text { MMSE }=22.1 \pm 4.7 \\
\text { CERAD }=1.3 \pm 1.8\end{array}$ & 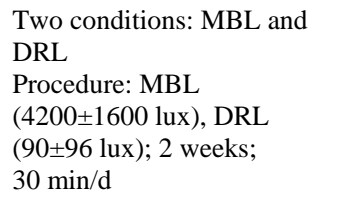 & Sleep & $\begin{array}{l}\text { Actigraphy, ESS, } \\
\text { Daily sleep } \\
\text { reports }\end{array}$ & 30-min MBL is not sufficient for improving sleep \\
\hline $\operatorname{Graf}(2001)^{34}$ & $\begin{array}{l}\text { Parallel group } \\
\text { design } \\
\text { Nursing home }\end{array}$ & $\begin{array}{l}n=23 \\
\text { Mean age }=82 . \\
1 \pm 6.4\end{array}$ & $\begin{array}{l}\text { AD or VD, mean MMSE: } \\
\text { MBL group }=15.2 \pm 4.8 \\
\text { MDL group }=17.1 \pm 7.1\end{array}$ & $\begin{array}{l}\text { Two conditions: MBL and } \\
\text { MDL. } \\
\text { Procedure: MBL ( } 3000 \text { lux), } \\
\text { MDL (100 lux); } 10 \mathrm{~d} ; 2 \text { h/d }\end{array}$ & Cognition & MMSE & $\begin{array}{l}\text { Significant beneficial effects on cognitive } \\
\text { functioning }\end{array}$ \\
\hline $\begin{array}{l}\text { Haffmans } \\
(2001)^{42}\end{array}$ & $\begin{array}{l}\text { Crossover } \\
\text { design Hospital }\end{array}$ & $\begin{array}{l}n=10(7 \text { women }) \\
\text { Mean age }=72.1\end{array}$ & Dementia diagnosis & $\begin{array}{l}\text { Two conditions: } \\
\text { MBL + melatonin and } \\
\text { MBL + placebo. } \\
\text { Procedure: } 10,000 \text { lux; } 2 \\
\text { periods of } 2 \text { weeks; } 30 \text { min/d }\end{array}$ & Agitation & CGI, GIP, SDAS & $\begin{array}{l}\text { Positive effects for the MBL combined with } \\
\text { placebo. Patients were less restless and more co- } \\
\text { operative. The condition with melatonin showed } \\
\text { no additional positive effects }\end{array}$ \\
\hline $\begin{array}{l}\text { Hickman } \\
(2007)^{40} \dagger\end{array}$ & $\begin{array}{l}\text { Cluster-unit } \\
\text { crossover design } \\
\text { Psychiatric } \\
\text { hospital and } \\
\text { nursing home }\end{array}$ & $\begin{array}{l}n=66(31 \text { women }) \\
\text { Mean age }=79\end{array}$ & $\begin{array}{l}\text { Dementia, MDS-COGS/ } \\
\text { MMSE: Mild to } \\
\text { moderate: } n=21 \text {; } \\
\text { severe: } n=31 \text {; and very } \\
\text { severe: } n=14\end{array}$ & $\begin{array}{l}\text { Four conditions: MBL, EBL, } \\
\text { all day BL, STL. } \\
\text { Procedure: BL (2000- } \\
3000 \text { lux), STL (500- } \\
600 \text { lux); } 3 \text { weeks; MBL and } \\
\text { EBL: } 4 \text { h/d, all day BL: } 13 \text { h/d }\end{array}$ & Mood & CSDD & $\begin{array}{l}\text { MBL significantly decreases depressive } \\
\text { symptoms in some people but may worsen } \\
\text { symptoms in others. Individual more than unit- } \\
\text { level BL interventions would be a more effective } \\
\text { strategy }\end{array}$ \\
\hline
\end{tabular}




\begin{tabular}{|c|c|c|c|c|c|c|c|}
\hline Author (year) & $\begin{array}{l}\text { Study design/ } \\
\text { Setting }\end{array}$ & Sample & Cognitive status & Intervention characteristics & $\begin{array}{l}\text { Outcome } \\
\text { measured }\end{array}$ & $\begin{array}{l}\text { Measurement } \\
\text { tools }\end{array}$ & Main findings \\
\hline $\begin{array}{l}\text { Lovell } \\
(1995)^{24}\end{array}$ & $\begin{array}{l}\text { ABABA design } \\
\text { Nursing home }\end{array}$ & $\begin{array}{l}n=6(5 \text { women }) \\
\text { Mean age }=89 . \\
2 \pm 3.4\end{array}$ & $\begin{array}{l}\text { Moderate to severe } \\
\text { dementia, mean MMSE }=8 \text {. } \\
7 \pm 5.2\end{array}$ & $\begin{array}{l}\text { One condition: MBL. } \\
\text { Procedure: } 2500 \text { lux; two } 10 \mathrm{~d} \\
\text { MBL periods; } 2 \mathrm{~h} / \mathrm{d}\end{array}$ & Agitation & ABRS & $\begin{array}{l}\text { Agitation is significantly lower on light treatment } \\
\text { days }\end{array}$ \\
\hline $\begin{array}{l}\text { Lyketsos } \\
(1999)^{63}\end{array}$ & $\begin{array}{l}\text { RCT } \\
\text { Nursing home }\end{array}$ & $\begin{array}{l}n=15(14 \text { women }) \\
\text { Mean age }=80 . \\
8 \pm 8.7\end{array}$ & $\begin{array}{l}\text { Dementia, mean } \\
\mathrm{MMSE}=6.4 \pm 6.8\end{array}$ & $\begin{array}{l}\text { Two conditions: MBL and } \\
\text { STL. } \\
\text { Procedure: MBL (10,000 lux); } \\
4 \text { weeks; } 1 \text { h/d }\end{array}$ & $\begin{array}{l}\text { Agitation, } \\
\text { Sleep, Mood }\end{array}$ & $\begin{array}{l}\text { BEHAVE-AD, } \\
\text { CSDD, Sleep } \\
\text { logs }\end{array}$ & $\begin{array}{l}\text { No effect on agitation or depression. Significant } \\
\text { improvement in nocturnal sleep }\end{array}$ \\
\hline $\begin{array}{l}\text { McCurry } \\
(2011)^{44}\end{array}$ & $\begin{array}{l}\text { RCT } \\
\text { Community- } \\
\text { dwelling }\end{array}$ & $\begin{array}{l}n=132(73 \\
\text { women) } \\
\text { Mean age }=81 . \\
0 \pm 8.0\end{array}$ & $\begin{array}{l}\text { Probable or possible AD } \\
\text { diagnosis, mean } \\
\text { MMSE }=18.7 \pm 6.9\end{array}$ & $\begin{array}{l}\text { Four conditions: 1-walking, 2- } \\
\text { EBL, 3- } \\
\text { walking + light + sleep } \\
\text { education, 4-control. } \\
\text { Procedure: EBL ( } 2500 \text { lux); } 8 \\
\text { weeks; } 1 \text { h/d }\end{array}$ & Sleep & Actigraphy, SDI & $\begin{array}{l}\text { Light therapy and walking, singly or in } \\
\text { combination, significantly improve night-time } \\
\text { sleep in individuals with sleep problems }\end{array}$ \\
\hline $\begin{array}{l}\text { Mishima } \\
(1994)^{35}\end{array}$ & $\begin{array}{l}\text { Two-group } \\
\text { pretest-posttest } \\
\text { design } \\
\text { Hospital }\end{array}$ & $\begin{array}{l}n=24 \\
\text { Mean age }=75\end{array}$ & $\begin{array}{l}\text { Moderate and severe } \\
\text { dementia, VD or AD } \\
\text { diagnosis }\end{array}$ & $\begin{array}{l}\text { Two conditions: MBL and } \\
\text { control. } \\
\text { Procedure: } 3000-5000 \text { lux; } 4 \\
\text { weeks; } 2 \text { h/d }\end{array}$ & Sleep, BPSD & $\begin{array}{l}\text { Sleep diary by } \\
\text { nursing staff }\end{array}$ & $\begin{array}{l}\text { Significant increase in total and nocturnal sleep } \\
\text { time and a significant decrease in daytime sleep } \\
\text { time. The presence of behaviour disorders during } \\
\text { the intervention period was significantly lower } \\
\text { than at baseline }\end{array}$ \\
\hline $\begin{array}{l}\text { Mishima } \\
(1998)^{36}\end{array}$ & $\begin{array}{l}\text { Crossover design } \\
\text { Hospital }\end{array}$ & $\begin{array}{l}n=22 \text { (9 women) } \\
\text { Mean age }=79.5\end{array}$ & $\begin{array}{l}\text { VD or AD diagnosis, mean } \\
\text { MMSE: VD group }=8.0 \\
\text { (range } 3-14) \text {; AD } \\
\text { group }=9.0 \text { (range } 3-17)\end{array}$ & $\begin{array}{l}\text { Two conditions: MBL and } \\
\text { MDL. } \\
\text { Procedure: MBL ( } 5000- \\
8000 \text { lux), MDL ( } 300 \text { lux); } 4 \\
\text { weeks; } 2 \text { h/d }\end{array}$ & Sleep & Actigraphy & $\begin{array}{l}\text { Significant improvement of MBL in nocturnal } \\
\text { sleep, reducing night-time activity, but only in the } \\
\text { VD group }\end{array}$ \\
\hline $\begin{array}{l}\text { Münch } \\
(2017)^{50}\end{array}$ & $\begin{array}{l}\text { Between-subject } \\
\text { study design } \\
\text { Nursing home }\end{array}$ & $\begin{array}{l}n=89 \text { (58 women) } \\
\text { Mean age }=78 . \\
4 \pm 9.0\end{array}$ & $\begin{array}{l}\text { Severe cognitive } \\
\text { impairment, mean S- } \\
\text { MMSE }=8.8 \pm 10.2\end{array}$ & $\begin{array}{l}\text { Two conditions: Low and high } \\
\text { light. } \\
\text { Procedure: Low (<417. } \\
24 \text { lux), high ( }>417.24 \text { lux);8 } \\
\text { weeks; } 10 \mathrm{~h} / \mathrm{d}\end{array}$ & $\begin{array}{l}\text { Emotions, } \\
\text { Agitation, } \\
\text { QoL, } \\
\text { Activity, } \\
\text { Sleep }\end{array}$ & $\begin{array}{l}\text { OERS, CADS, } \\
\text { CMAI, } \\
\text { QUALID, Wrist- } \\
\text { worn activity } \\
\text { watch }\end{array}$ & $\begin{array}{l}\text { Higher light exposure results in an increase in } \\
\text { positive emotions, greater general alertness and } \\
\text { better quality of life }\end{array}$ \\
\hline $\begin{array}{l}\text { Onega } \\
(2016)^{53}\end{array}$ & $\begin{array}{l}\text { RCT } \\
\text { Nursing home }\end{array}$ & $\begin{array}{l}n=60 \text { ( } 43 \text { women }) \\
\text { Mean age }=82 . \\
6 \pm 9.6\end{array}$ & $\begin{array}{l}\text { Dementia diagnosis, } \\
\text { Mild: } n=7 ; \\
\text { Moderate: } n=11 \text {; } \\
\text { Severe: } n=42 . ; \text { mean } \\
\text { MMSE }=7.2 \pm 6.9\end{array}$ & $\begin{array}{l}\text { Two conditions: MBL+EBL } \\
\text { and STL. } \\
\text { Procedure: BL (10,000 lux), } \\
\text { STL ( } 250 \text { lux); } 8 \text { weeks; } \\
30 \text { min twice a day; } 5 \text { d/week }\end{array}$ & $\begin{array}{l}\text { Mood, } \\
\text { Agitation }\end{array}$ & $\begin{array}{l}\text { DSAOA, } \\
\text { DMAS-17, } \\
\text { CSDD, PAS } \\
\text { BARS, CMAI-F, } \\
\text { CMAI-D }\end{array}$ & $\begin{array}{l}\text { Significant improvement in all three measures of } \\
\text { depression and all four measures of agitation }\end{array}$ \\
\hline
\end{tabular}




\begin{tabular}{|c|c|c|c|c|c|c|c|}
\hline Author (year) & $\begin{array}{l}\text { Study design/ } \\
\text { Setting }\end{array}$ & Sample & Cognitive status & Intervention characteristics & $\begin{array}{l}\text { Outcome } \\
\text { measured }\end{array}$ & $\begin{array}{l}\text { Measurement } \\
\text { tools }\end{array}$ & Main findings \\
\hline $\begin{array}{l}\text { Onega } \\
(2018)^{67} \boldsymbol{q}\end{array}$ & $\begin{array}{l}\text { Mix-model } \\
\text { repeated } \\
\text { measures } \\
\text { Nursing home }\end{array}$ & $\begin{array}{l}n=60 \text { ( } 43 \text { women }) \\
\text { Mean age }=82 . \\
6 \pm 9.6\end{array}$ & $\begin{array}{l}\text { Dementia diagnosis, } \\
\text { Mild/moderate: } n=18 \text {; } \\
\text { Severe: } n=42 ; \text { mean } \\
\text { MMSE }=7.2 \pm 6.9\end{array}$ & $\begin{array}{l}\text { Two conditions: MBL+EBL } \\
\text { and STL. } \\
\text { Procedure: BL (10,000 lux), } \\
\text { STL (250 lux); } 8 \text { weeks; } \\
30 \text { min twice a day; } 5 \text { d/week }\end{array}$ & Mood & $\begin{array}{l}\text { DSAOA, CSDD } \\
\text { (Analysis of the } \\
\text { total scores and } \\
\text { their individual } \\
\text { subscales) }\end{array}$ & $\begin{array}{l}\text { Total scores: equally effective at mild/moderate } \\
\text { and severe stages of dementia. Subscales: greater } \\
\text { benefits for severe dementia than mild/moderate } \\
\text { in two DSAOA subscales (disagreeable behaviour } \\
\text { and sleep impairment) }\end{array}$ \\
\hline $\begin{array}{l}\text { Riemersma- } \\
\text { van der Lek } \\
(2008)^{30}\end{array}$ & $\begin{array}{l}\text { RCT } \\
\text { Assisted care } \\
\text { facilities }\end{array}$ & $\begin{array}{l}n=189(170 \\
\text { woman) } \\
\text { Mean age }=85 \\
5 \pm 5.5\end{array}$ & $\begin{array}{l}\text { Dementia diagnosis, mean } \\
\text { MMSE }=16.4 \pm 5.6\end{array}$ & $\begin{array}{l}\text { Four conditions: 1-BL, 2- } \\
\text { Melatonin, 3-BL + Melatonin, } \\
\text { 4-DL. } \\
\text { Procedure: BL ( } \pm 1000 \text { lux), } \\
\text { DL ( } \pm 300 \text { lux); } 15 \pm 12 \\
\text { months; } 9 \text { h/d. }\end{array}$ & $\begin{array}{l}\text { Cognition, } \\
\text { Mood, } \\
\text { Sleep, } \\
\text { Agitation, } \\
\text { NPS, ADL }\end{array}$ & $\begin{array}{l}\text { MMSE, CSDD, } \\
\text { PGCMS, } \\
\text { PGCARS, } \\
\text { MOSES, CMAI, } \\
\text { NI-ADL, NPI-Q } \\
\text { Actigraphy }\end{array}$ & $\begin{array}{l}\text { BL ameliorated cognition and depressive } \\
\text { symptoms and also attenuated the increase in } \\
\text { functional limitations over time. Melatonin + LB } \\
\text { improved agitation, nocturnal restlessness, sleep } \\
\text { efficiency, duration and fragmentation }\end{array}$ \\
\hline $\begin{array}{l}\text { Satlin } \\
(1992)^{37}\end{array}$ & $\begin{array}{l}\text { Open clinical } \\
\text { trial } \\
\text { Hospital }\end{array}$ & $\begin{array}{l}n=10(1 \text { woman }) \\
\text { Mean age }=70 \\
1 \pm 5.1\end{array}$ & $\begin{array}{l}\text { Moderate to severe } \\
\text { dementia, mean MMSE }=0 \text {. } \\
6 \pm 1.1\end{array}$ & $\begin{array}{l}\text { One condition: MBL. } \\
\text { Procedure: 1500-2000 lux; } 2 \\
\text { weeks; } 2 \text { h/d }\end{array}$ & $\begin{array}{l}\text { Agitation, } \\
\text { Sleep }\end{array}$ & $\begin{array}{l}\text { Daily ratings by } \\
\text { nursing staff }\end{array}$ & $\begin{array}{l}\text { Improvements in sleep-wakefulness, nocturnal } \\
\text { activity decreased and the relative amplitude of } \\
\text { circadian rhythm increased. No effects on } \\
\text { agitation }\end{array}$ \\
\hline $\begin{array}{l}\text { Schindler } \\
(2002)^{29}\end{array}$ & $\begin{array}{l}\text { Case series } \\
\text { Hospital }\end{array}$ & $\begin{array}{l}n=5(4 \text { women }) \\
\text { Mean age }=81 . \\
8 \pm 6.3\end{array}$ & Dementia diagnosis & $\begin{array}{l}\text { One condition: MBL } \\
\text { Procedure: } 2500 \text { lux; } 2 \text { weeks; } \\
2 \text { h/d }\end{array}$ & NPS & CRS & $\begin{array}{l}\text { Delusions slightly improved in three of the } \\
\text { participants, another did not show them neither } \\
\text { before or during MBL, and another developed } \\
\text { delirious symptomatology }\end{array}$ \\
\hline $\begin{array}{l}\text { Sekiguchi } \\
(2017)^{38}\end{array}$ & $\begin{array}{l}\text { Case series } \\
\text { Hospital }\end{array}$ & $\begin{array}{l}n=17 \text { ( } 6 \text { women }) \\
\text { Mean age }=75 \\
5 \pm 6.2\end{array}$ & $\begin{array}{l}\text { Dementia diagnosis, mean } \\
\text { MMSE }=12.1 \pm 8.2\end{array}$ & $\begin{array}{l}\text { One condition: MBL. } \\
\text { Procedure: } 5000 \text { lux; } 2 \text { weeks; } \\
1 \text { h/d }\end{array}$ & Sleep & $\begin{array}{l}\text { NPI-NH (Sleep } \\
\text { disturbances) }\end{array}$ & $\begin{array}{l}\text { Improvement of sleep disturbance in four AD } \\
\text { patients. No significant values provided }\end{array}$ \\
\hline $\begin{array}{l}\text { Skjerve } \\
(2004)^{39}\end{array}$ & $\begin{array}{l}\text { Open clinical } \\
\text { trial } \\
\text { Nursing home }\end{array}$ & $\begin{array}{l}n=10 \text { ( } 3 \text { women }) \\
\text { Mean age }=79.4\end{array}$ & $\begin{array}{l}\text { VD or AD diagnosis, } \\
\text { Median MMSE }=0 \\
(\text { range }=0-11 \text { ) }\end{array}$ & $\begin{array}{l}\text { One condition: MBL. } \\
\text { Procedure: 5000-8000 lux; } 4 \\
\text { weeks; } 45 \mathrm{~min} / \mathrm{d}\end{array}$ & BPSD, Sleep & $\begin{array}{l}\text { CMAI, } \\
\text { BEHAVE-AD, } \\
\text { Own scale for } \\
\text { sleep-wake } \\
\text { disturbances, } \\
\text { Actigraphy }\end{array}$ & $\begin{array}{l}\text { Significant reduction of BPSD. No effect on } \\
\text { sleep-wake measures but an improvement of } \\
\text { activity rhythm disturbances }\end{array}$ \\
\hline $\begin{array}{l}\text { Sloane } \\
(2007)^{41} \dagger\end{array}$ & $\begin{array}{l}\text { Cluster-unit } \\
\text { crossover design } \\
\text { Psychiatric } \\
\text { hospital and } \\
\text { nursing home }\end{array}$ & $\begin{array}{l}n=66 \text { ( } 31 \text { women) } \\
\text { Mean age }=79\end{array}$ & $\begin{array}{l}\text { Dementia, MDS-COGS/ } \\
\text { MMSE: Mild to } \\
\text { moderate: } n=21 \text {; } \\
\text { severe: } n=31 \text {; and very } \\
\text { severe: } n=14\end{array}$ & $\begin{array}{l}\text { Four conditions: MBL, EBL, } \\
\text { all day BL, STL. } \\
\text { Procedure: BL ( } 2000- \\
3000 \text { lux), STL (500- } \\
600 \text { lux); } 3 \text { weeks; MBL and } \\
\text { EBL: } 4 \text { h/d, all day BL: } 13 \text { h/d }\end{array}$ & $\begin{array}{l}\text { Sleep, } \\
\text { Circadian } \\
\text { rhythm }\end{array}$ & Actigraphy & $\begin{array}{l}\text { Night-time sleep increased significantly in } \\
\text { participants exposed to morning and all-day light, } \\
\text { with the increase most prominent in participants } \\
\text { with severe or very severe dementia }\end{array}$ \\
\hline
\end{tabular}




\begin{tabular}{|c|c|c|c|c|c|c|c|}
\hline Author (year) & $\begin{array}{l}\text { Study design/ } \\
\text { Setting }\end{array}$ & Sample & Cognitive status & Intervention characteristics & $\begin{array}{l}\text { Outcome } \\
\text { measured }\end{array}$ & $\begin{array}{l}\text { Measurement } \\
\text { tools }\end{array}$ & Main findings \\
\hline $\begin{array}{l}\text { Sloane } \\
(2015)^{45}\end{array}$ & $\begin{array}{l}\text { RCT } \\
\text { Community- } \\
\text { dwelling }\end{array}$ & $\begin{array}{l}n=17(11 \\
\text { women); } \\
\text { Age: } 65-79=6 \\
\geq 80=11\end{array}$ & $\begin{array}{l}\text { Dementia diagnosis; mean } \\
\mathrm{MMSE}=12.7 \pm 9.1\end{array}$ & $\begin{array}{l}\text { Two conditions: BWL and } \\
\text { RYL. } \\
\text { Procedure: BWL ( } 300- \\
400 \text { lux), RYL } \\
\text { (mean = } 118 \text { lux); } 6 \text { weeks; } \\
\text { from awakening time until } \\
\text { 18:00 }\end{array}$ & $\begin{array}{l}\text { Sleep, } \\
\text { Mood, } \\
\text { QoL }\end{array}$ & $\begin{array}{l}\text { Actigraphy, } \\
\text { PSQI, MOS, } \\
\text { ESS, CSDD, } \\
\text { PHQ-9, QOL- } \\
\text { AD }\end{array}$ & $\begin{array}{l}\text { No significant evidence of sleep improvement or } \\
\text { other outcomes in people with dementia. For } \\
\text { caregivers, a significant improvement in sleep was } \\
\text { observed }\end{array}$ \\
\hline $\begin{array}{l}\text { Thorpe } \\
(2000)^{52}\end{array}$ & $\begin{array}{l}\text { ABA repeated } \\
\text { measures } \\
\text { Long term care } \\
\text { facility }\end{array}$ & $\begin{array}{l}n=16(13 \text { women }) \\
\text { Mean age }=80.3\end{array}$ & $\begin{array}{l}\text { Dementia diagnosis; mean } \\
\text { GDS = } 6 \text { (range 5-7) }\end{array}$ & $\begin{array}{l}\text { One condition: MBL. } \\
\text { Procedure: } 10,000 \text { lux; } 1 \\
\text { week; } 90 \mathrm{~min} / \mathrm{d} \text { (Monday to } \\
\text { Friday) }\end{array}$ & $\begin{array}{l}\text { Agitation, } \\
\text { BPSD }\end{array}$ & CMAI, EBIC & $\begin{array}{l}\text { CMAI: a significant decrease in agitation during } \\
\text { MBL compared with the post-therapy week. } \\
\text { EBIC: MBL showed a trend to increase positive } \\
\text { behaviours and to decrease disruptive behaviours }\end{array}$ \\
\hline $\begin{array}{l}\text { Van Someren } \\
(1997)^{43}\end{array}$ & $\begin{array}{l}\text { ABA Repeated } \\
\text { measures } \\
\text { Psychogeriatric } \\
\text { ward }\end{array}$ & $\begin{array}{l}n=22(15 \text { women }) \\
\text { Mean age }=79 \pm 2\end{array}$ & $\begin{array}{l}\text { Dementia diagnosis; mean } \\
\text { GDS }=6.3 \pm 0.13 \text { (range } \\
\text { 5-7) }\end{array}$ & $\begin{array}{l}\text { Two condition: BL and } \\
\text { baseline. } \\
\text { Procedure: BL } \\
\text { (mean = } 1136 \pm 89 \text { lux), } \\
\text { baseline (mean = 436 } \pm 90 \text { or } \\
372 \pm 89 \text { lux); } 4 \text { weeks; all-day }\end{array}$ & $\begin{array}{l}\text { Circadian } \\
\text { rhythm }\end{array}$ & Actigraphy & $\begin{array}{l}\text { BL improves the circadian rest-activity rhythm } \\
\text { disturbances in severely demented patients with } \\
\text { intact vision, but not in those with visual } \\
\text { impairment }\end{array}$ \\
\hline
\end{tabular}

Note. *, †, $\ddagger$, §, ๆ: Articles with the same symbol report data from the same study sample but describe different outcomes. Abbreviations: ABA: baseline with no treatment (A) and treatment (B); ABABA: Alternating baseline (A) and treatment (B) conditions; ABRS: Agitated Behaviour Rating Scale; AD: Alzheimer's Disease; ADL: Activities of Daily Living; BARS: Brief Agitation Rating Scale; BEHAVE-AD: The Behavioural pathology in Alzheimer's Disease; BIMS: Brief Interview for Mental Status; BL: Bright Light; BPSD: Behavioural and Psychological Symptoms of Dementia; BWL: Blue-White Light; CADS: Change in Advanced Dementia Score; CDR: Clinical Dementia Rating; CERAD: Consortium to Establish a Registry for Alzheimer's Disease; CGI: Clinical Global Impression; CMAI: Cohen-Mansfield Agitation Inventory; CMAI-D: Cohen-Mansfield Agitation Inventory-Disruptiveness; CMAI-F: Cohen-Mansfield Agitation Inventory-Frequency; CRBRS: Crichton Royal Behaviour Rating Scale; CRS: Confusion Rating Scale; CSDD: Cornell Scale for Depression in Dementia; DDS: Dawn-Dusk Simulation; DL: Dim light; DMAS-17: Dementia Mood Assessment Scale-17 item; DRL: Dim Red Light; DSAOA: Depressive Symptom Assessment in Older Adults; EBIC: Environment-Behaviour Interaction Code; EBL: Evening Bright Light; ESS Epworth Sleepiness Scale; GDS: Global Deterioration Scale; GDS-SF: Geriatric Depression Scale - Short Form; GIP: Gedragsobservatieschaal voor Intramurale Psychogeriatrie; MBL: Morning Bright Light; MD: Mixed Dementia; MDL: Morning Dim Light; MDS-ADL: Minimum Data Set - Activities of Daily Living Scale; MDS-COGS: Minimum Data Set - Cognition Scale; MMSE: MiniMental State Examination; MOS: Medical Outcomes Study sleep scale; MOSES: Multi Observation Scale for Elderly Subjects; MOUSEPAD: Manchester and Oxford Universities Scale for the Psychopathological Assessment of Dementia; NI-ADL: Nurse-informant activities of daily living scale; NPI-NH: Neuropsychiatric Inventory Nursing Home Version; NPS: Neuropsychiatric Symptoms; OERS: Observed Emotion Rating Scale; PAS: Pittsburgh Agitation Scale; NPI-Q: Questionnaire format of Neuropsychiatric Inventory; PGCARS: Philadelphia Geriatric Centre Affect Rating Scale; PGCMS: Philadelphia Geriatric Centre Morale Scale; PHQ-9: Patient Health Questionnaire; PSQI: Pittsburgh Sleep Quality Index; QoL: Quality of Life; QOL_AD: Quality of Life in Alzheimer's Disease; QUALID: Quality of Life for Severe Dementia; RCT: Randomized Controlled Trial; RYL: Red-Yellow Light; SDAS: Social Dysfunction and Aggression Scale; SDI: Sleep Disorders Inventory; S-MMSE: Severe Mini- Mental State Examination; STL: Standard Light; VD: Vascular Dementia. 
In this systematic review, the mean sample size was $42.5 \pm 42.5$ in 29 studies (36 articles), with a minimum of 5 individuals ${ }^{29}$ and a maximum of $189 .^{30}$ For all studies included in the analysis, the total sample size was 1233 older adults (66.1\% women). Only nine articles contained a greater

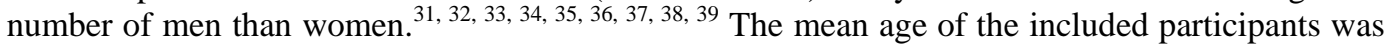
$81.3 \pm 4.5$ years, ranging from $70.1 \pm 5.1^{37}$ to $89.2 \pm 3.4$ years.24 Most of the articles assessed the effect of light therapy in older adults within the residential/nursing home setting, excluding three that also combined participants from hospitals, ${ }^{31,40,41}$ seven that only involved a hospital setting29, ${ }^{35,36,37,38,42,43}$ and four that included community-dwelling older adults. ${ }^{32,33,44,45}$ Twenty of the articles identified in the search were from the USA, 4 from Norway, ${ }^{39,46,47,48}$ three from Japan, ${ }^{35,36,38}$ three from the Netherlands, ${ }^{30,42,43}$ two from Austria, ${ }^{29,34}$ two from Switzerland, ${ }^{49,50}$ one from the United Kingdom, ${ }^{51}$ and one from Canada. ${ }^{52}$

The results are grouped into six subsections: the first one includes the methodological quality assessment of included articles. The following 4 subsections present the main outcomes (light therapy and BPSD, light therapy and cognition, light therapy and quality of life, and light therapy and activities of daily living) described in Table 1 . As observed, three of the articles examined the effect of light treatment on BPSD as a whole, regarding effects on specific BPSD: 25 of the articles examined the effect of light on sleep, 12 on agitation, 10 on mood and 4 on neuropsychiatric symptoms. Additionally, four articles assessed the effect on cognition, two on quality of life and two on functioning in activities of daily living. A final sixth subsection was included to compare the intervention conditions of light therapy among the studies analysed in this review.

\section{Quality assessment}

Regarding quality assessment, studies were analysed using the corresponding form of JBI appraisal tool according to the type of design used in each article: checklist for Randomized Controlled Trials - RCT (Table 2) and checklist for Quasi-Experimental Studies - QES (Table 3). In Table 2, only three $e^{30,44,53}$ of the 18 included studies met 10 or more $(80 \%)$ of the 13 criteria on the JBI for RCT. A lack of quality was observed in most of the 18 studies, 10 (55.6\%) of them ${ }^{19,33,34,45,49,54,55,56,57,58}$ had equal or below six scores (less than half of the defined appraisal criteria). Category 12 (appropriate statistical analysis) was the most unfulfilled item, no power analysed or effect sizes were estimated. In category 9 (participants analysed in the original group) was rated as not applicable (N/A) in two of the included studies ${ }^{19,59}$ since their follow-up was complete, so the intention-to-treat analysis was not used in these cases. Randomization (Q1), identical group treatment (Q7), complete follow-up (Q8), outcomes measured (Q10), and trial design (Q13) were the best methodological standards. Methodological quality was better in those non-randomized studies (Table 3), all 18 studies met at least 5 (50\%) of the defined appraisal criteria. Four $^{31,40,41,50}$ of the 18 assessed articles met 7 or more $(80 \%)$ of the 9 criteria of the JBI for QES. Categories 4 (presence of a control group) and 9 (appropriate statistical analysis) was the most unfulfilled item, no power analysed or effect sizes were estimated. A clear cause/effect (Q1), identical compared groups (Q2), pre- and post- measures (Q5), complete follow-up (Q6), and outcomes measured (Q7) were the most covered items for non-randomized experimental studies. 
Table 2. Methodological quality assessment using The Joanna Briggs Institute Critical Appraisal Checklist for Randomized Clinical Trials.

\begin{tabular}{|c|c|c|c|c|c|c|c|c|c|c|c|c|c|c|}
\hline Study & Q1 & Q2 & Q3 & Q4 & Q5 & Q6 & Q7 & Q8 & Q9 & Q10 & Q11 & Q12 & Q13 & Score $(/ 13)$ \\
\hline Ancoli-Israel et al. ${ }^{54}$ & $\mathrm{Y}$ & $\mathrm{U}$ & $\mathrm{N}$ & $\mathrm{U}$ & $\mathrm{U}$ & $\mathrm{N}$ & $\mathrm{Y}$ & $\mathrm{N}$ & $\mathrm{N}$ & $\mathrm{Y}$ & $\mathrm{U}$ & $\mathrm{N}$ & $\mathrm{Y}$ & 4 \\
\hline Ancoli-Israel et al. ${ }^{55}$ & $\mathrm{Y}$ & $\mathrm{U}$ & $\mathrm{N}$ & $\mathrm{U}$ & $\mathrm{N}$ & $\mathrm{N}$ & $\mathrm{Y}$ & $\mathrm{N}$ & $\mathrm{U}$ & $\mathrm{Y}$ & $\mathrm{Y}$ & $\mathrm{N}$ & $\mathrm{Y}$ & 5 \\
\hline Ancoli-Israel et al. ${ }^{56}$ & $\mathrm{Y}$ & $\mathrm{U}$ & $\mathrm{N}$ & $\mathrm{U}$ & $\mathrm{N}$ & $\mathrm{N}$ & $\mathrm{Y}$ & $\mathrm{Y}$ & $\mathrm{U}$ & $\mathrm{Y}$ & $\mathrm{Y}$ & $\mathrm{N}$ & $\mathrm{Y}$ & 6 \\
\hline Burns et al. ${ }^{51}$ & $\mathrm{Y}$ & $\mathrm{U}$ & $\mathrm{Y}$ & $\mathrm{U}$ & $\mathrm{U}$ & $\mathrm{Y}$ & $\mathrm{Y}$ & $\mathrm{Y}$ & $\mathrm{Y}$ & $\mathrm{Y}$ & $\mathrm{Y}$ & $\mathrm{N}$ & $\mathrm{Y}$ & 9 \\
\hline Dowling et al. ${ }^{19}$ & $\mathrm{Y}$ & $\mathrm{U}$ & $\mathrm{Y}$ & $\mathrm{N}$ & $\mathrm{N}$ & $\mathrm{N}$ & $\mathrm{Y}$ & $\mathrm{Y}$ & N/A & $\mathrm{Y}$ & $\mathrm{U}$ & $\mathrm{N}$ & $\mathrm{Y}$ & 6 \\
\hline Dowling et al. ${ }^{57}$ & $\mathrm{Y}$ & $\mathrm{U}$ & $\mathrm{Y}$ & $\mathrm{N}$ & $\mathrm{N}$ & $\mathrm{N}$ & $\mathrm{Y}$ & $\mathrm{Y}$ & $\mathrm{U}$ & $\mathrm{Y}$ & $\mathrm{U}$ & $\mathrm{N}$ & $\mathrm{Y}$ & 6 \\
\hline Dowling et al. ${ }^{58}$ & $\mathrm{Y}$ & $\mathrm{U}$ & $\mathrm{Y}$ & $\mathrm{N}$ & $\mathrm{N}$ & $\mathrm{N}$ & $\mathrm{Y}$ & $\mathrm{U}$ & $\mathrm{U}$ & $\mathrm{Y}$ & $\mathrm{Y}$ & $\mathrm{N}$ & $\mathrm{Y}$ & 6 \\
\hline Dowling et al. ${ }^{59}$ & $\mathrm{Y}$ & $\mathrm{U}$ & $\mathrm{N}$ & $\mathrm{Y}$ & $\mathrm{Y}$ & $\mathrm{Y}$ & $\mathrm{Y}$ & $\mathrm{Y}$ & N/A & $\mathrm{Y}$ & $\mathrm{U}$ & $\mathrm{Y}$ & $\mathrm{Y}$ & 9 \\
\hline Fontana Gasio et al. ${ }^{49}$ & $\mathrm{Y}$ & $\mathrm{U}$ & $\mathrm{Y}$ & $\mathrm{U}$ & $\mathrm{U}$ & $\mathrm{U}$ & $\mathrm{U}$ & $\mathrm{Y}$ & $\mathrm{U}$ & $\mathrm{Y}$ & $\mathrm{U}$ & $\mathrm{Y}$ & $\mathrm{Y}$ & 6 \\
\hline Friedman et al. ${ }^{33}$ & $\mathrm{Y}$ & $\mathrm{U}$ & $\mathrm{Y}$ & $\mathrm{U}$ & $\mathrm{U}$ & $\mathrm{U}$ & $\mathrm{Y}$ & $\mathrm{Y}$ & $\mathrm{U}$ & $\mathrm{Y}$ & $\mathrm{U}$ & $\mathrm{N}$ & $\mathrm{Y}$ & 6 \\
\hline Graf et al. ${ }^{34}$ & $\mathrm{Y}$ & $\mathrm{U}$ & $\mathrm{U}$ & $\mathrm{U}$ & $\mathrm{U}$ & $\mathrm{Y}$ & $\mathrm{U}$ & $\mathrm{Y}$ & $\mathrm{U}$ & $\mathrm{Y}$ & $\mathrm{U}$ & $\mathrm{N}$ & $\mathrm{Y}$ & 5 \\
\hline Haffmans et al. ${ }^{42}$ & $\mathrm{Y}$ & $\mathrm{U}$ & $\mathrm{U}$ & $\mathrm{Y}$ & $\mathrm{Y}$ & $\mathrm{Y}$ & $\mathrm{U}$ & $\mathrm{Y}$ & $\mathrm{U}$ & $\mathrm{Y}$ & $\mathrm{Y}$ & $\mathrm{N}$ & $\mathrm{Y}$ & 8 \\
\hline Lyketsos et al. ${ }^{63}$ & $\mathrm{Y}$ & $\mathrm{U}$ & $\mathrm{Y}$ & $\mathrm{U}$ & $\mathrm{U}$ & $\mathrm{Y}$ & $\mathrm{Y}$ & $\mathrm{Y}$ & $\mathrm{Y}$ & $\mathrm{Y}$ & $\mathrm{U}$ & $\mathrm{N}$ & $\mathrm{Y}$ & 8 \\
\hline McCurry et al. ${ }^{44}$ & $\mathrm{Y}$ & $\mathrm{Y}$ & $\mathrm{Y}$ & $\mathrm{U}$ & $\mathrm{U}$ & $\mathrm{Y}$ & $\mathrm{Y}$ & $\mathrm{Y}$ & $\mathrm{Y}$ & $\mathrm{Y}$ & $\mathrm{Y}$ & $\mathrm{Y}$ & $\mathrm{Y}$ & 11 \\
\hline Onega et al. ${ }^{53}$ & $\mathrm{Y}$ & $\mathrm{U}$ & $\mathrm{Y}$ & $\mathrm{Y}$ & $\mathrm{N}$ & $\mathrm{Y}$ & $\mathrm{Y}$ & $\mathrm{Y}$ & $\mathrm{U}$ & $\mathrm{Y}$ & $\mathrm{Y}$ & $\mathrm{Y}$ & $\mathrm{Y}$ & 10 \\
\hline Onega et al. ${ }^{67}$ & $\mathrm{Y}$ & $\mathrm{U}$ & $\mathrm{N}$ & $\mathrm{Y}$ & $\mathrm{N}$ & $\mathrm{Y}$ & $\mathrm{Y}$ & $\mathrm{Y}$ & $\mathrm{U}$ & $\mathrm{Y}$ & $\mathrm{Y}$ & $\mathrm{N}$ & $\mathrm{Y}$ & 9 \\
\hline Riemersma van der Lek et al. ${ }^{30}$ & $\mathrm{Y}$ & $\mathrm{Y}$ & $\mathrm{N}$ & $\mathrm{Y}$ & $\mathrm{Y}$ & $\mathrm{Y}$ & $\mathrm{Y}$ & $\mathrm{Y}$ & $\mathrm{Y}$ & $\mathrm{Y}$ & $\mathrm{U}$ & $\mathrm{Y}$ & $\mathrm{Y}$ & 11 \\
\hline Sloane et al. ${ }^{45}$ & $\mathrm{Y}$ & $\mathrm{U}$ & $\mathrm{U}$ & $\mathrm{U}$ & $\mathrm{U}$ & $\mathrm{U}$ & $\mathrm{U}$ & $\mathrm{Y}$ & $\mathrm{U}$ & $\mathrm{Y}$ & $\mathrm{U}$ & $\mathrm{N}$ & $\mathrm{Y}$ & 4 \\
\hline
\end{tabular}

Note: $\mathrm{Q}_{1}$ : Randomization; $\mathrm{Q}_{2}$ : Concealed allocation; $\mathrm{Q}_{3}$ : Groups similar at baseline; $\mathrm{Q}_{4}$ : Participants blinded; $\mathrm{Q}_{5}$ : Allocator blinded; $\mathrm{Q}_{6}$ : Assessors blinded; $\mathrm{Q}_{7}$ : Groups treated equally; $\mathrm{Q}_{8}$ : Follow-up complete; $\mathrm{Q}_{9}$ : Participants analysed in original group; $\mathrm{Q}_{10}$ : Outcomes measured in the same way in groups; $\mathrm{Q}_{11}$ : Outcomes measured reliably; $\mathrm{Q}_{12}$ : Appropriate statistical analysis; $\mathrm{Q}_{13}$ : Appropriate trial design. Abbreviations: Y: Yes; N: No; U: Unclear; N/A: not applicable.

\section{Light therapy and BPSD}

Three studies ${ }^{35,39,52}$ analysing the effect of light therapy on BPSD as a whole found improvements on BPSD. Two of these $\mathrm{e}^{35,52}$ showed a substantial reduction in the frequency of behaviour disorders, and in one of them, ${ }^{52}$ it was also reported a mean decrease of total disruptive behaviours during the light intervention.

In the following subsections, our results are presented classified according to the effect of light therapy on specific BPSD: sleep, agitation, mood, and neuropsychiatric symptoms. 
Table 3. Methodological quality assessment using The Joanna Briggs Institute Critical Appraisal Checklist for QuasiExperimental Studies.

\begin{tabular}{|c|c|c|c|c|c|c|c|c|c|c|}
\hline Study & Q1 & Q2 & Q3 & Q4 & Q5 & Q6 & Q7 & Q8 & Q9 & Score (/9) \\
\hline Barrick et al. ${ }^{31}$ & $\mathrm{Y}$ & $\mathrm{Y}$ & $\mathrm{U}$ & $\mathrm{Y}$ & $\mathrm{Y}$ & $\mathrm{Y}$ & $\mathrm{Y}$ & $\mathrm{Y}$ & $\mathrm{Y}$ & 8 \\
\hline Fetveit et al. ${ }^{46}$ & $\mathrm{Y}$ & $\mathrm{Y}$ & $\mathrm{U}$ & $\mathrm{N}$ & $\mathrm{Y}$ & $\mathrm{Y}$ & $\mathrm{Y}$ & $\mathrm{U}$ & $\mathrm{N}$ & 5 \\
\hline Fetveit and Bjorvatn ${ }^{47}$ & $\mathrm{Y}$ & $\mathrm{Y}$ & $\mathrm{U}$ & $\mathrm{N}$ & $\mathrm{Y}$ & $\mathrm{Y}$ & $\mathrm{Y}$ & $\mathrm{Y}$ & $\mathrm{N}$ & 6 \\
\hline Fetveit and Bjorvatn ${ }^{48}$ & $\mathrm{Y}$ & $\mathrm{Y}$ & $\mathrm{U}$ & $\mathrm{N}$ & $\mathrm{Y}$ & $\mathrm{Y}$ & $\mathrm{Y}$ & $\mathrm{Y}$ & $\mathrm{N}$ & 6 \\
\hline Figueiro et al. ${ }^{64}$ & $\mathrm{Y}$ & $\mathrm{Y}$ & $\mathrm{U}$ & $\mathrm{N}$ & $\mathrm{Y}$ & $\mathrm{N}$ & $\mathrm{Y}$ & $\mathrm{Y}$ & $\mathrm{N}$ & 5 \\
\hline Figueiro et al. $^{32}$ & $\mathrm{Y}$ & $\mathrm{Y}$ & $\mathrm{U}$ & $\mathrm{N}$ & $\mathrm{Y}$ & $\mathrm{N}$ & $\mathrm{Y}$ & $\mathrm{Y}$ & $\mathrm{Y}$ & 6 \\
\hline Hickman et al. ${ }^{40}$ & $\mathrm{Y}$ & $\mathrm{Y}$ & $\mathrm{U}$ & $\mathrm{Y}$ & $\mathrm{Y}$ & $\mathrm{Y}$ & $\mathrm{Y}$ & $\mathrm{Y}$ & $\mathrm{Y}$ & 8 \\
\hline Lovell et al. ${ }^{24}$ & $\mathrm{Y}$ & $\mathrm{Y}$ & $\mathrm{U}$ & $\mathrm{N}$ & $\mathrm{Y}$ & $\mathrm{Y}$ & $\mathrm{Y}$ & $\mathrm{U}$ & $\mathrm{N}$ & 5 \\
\hline Mishima et al. ${ }^{35}$ & $\mathrm{Y}$ & $\mathrm{N}$ & $\mathrm{U}$ & $\mathrm{Y}$ & $\mathrm{Y}$ & $\mathrm{Y}$ & $\mathrm{Y}$ & $\mathrm{Y}$ & $\mathrm{N}$ & 6 \\
\hline Mishima et al. ${ }^{36}$ & $\mathrm{Y}$ & $\mathrm{N}$ & $\mathrm{U}$ & $\mathrm{N}$ & $\mathrm{Y}$ & $\mathrm{Y}$ & $\mathrm{Y}$ & $\mathrm{Y}$ & $\mathrm{N}$ & 5 \\
\hline Münch et al. ${ }^{50}$ & $\mathrm{Y}$ & $\mathrm{Y}$ & $\mathrm{U}$ & $\mathrm{Y}$ & $\mathrm{N}$ & $\mathrm{Y}$ & $\mathrm{Y}$ & $\mathrm{Y}$ & $\mathrm{Y}$ & 7 \\
\hline Satlin et al. ${ }^{37}$ & $\mathrm{Y}$ & $\mathrm{Y}$ & $\mathrm{U}$ & $\mathrm{N}$ & $\mathrm{Y}$ & $\mathrm{Y}$ & $\mathrm{Y}$ & $\mathrm{U}$ & $\mathrm{N}$ & 5 \\
\hline Schindler et al. ${ }^{29}$ & $\mathrm{Y}$ & $\mathrm{Y}$ & $\mathrm{U}$ & $\mathrm{N}$ & $\mathrm{Y}$ & $\mathrm{Y}$ & $\mathrm{Y}$ & $\mathrm{U}$ & $\mathrm{N}$ & 5 \\
\hline Sekiguchi et al. ${ }^{38}$ & $\mathrm{Y}$ & $\mathrm{Y}$ & $\mathrm{U}$ & $\mathrm{N}$ & $\mathrm{Y}$ & $\mathrm{Y}$ & $\mathrm{Y}$ & $\mathrm{U}$ & $\mathrm{N}$ & 5 \\
\hline Skjerve et al. ${ }^{39}$ & $\mathrm{Y}$ & $\mathrm{Y}$ & $\mathrm{U}$ & $\mathrm{N}$ & $\mathrm{Y}$ & $\mathrm{Y}$ & $\mathrm{Y}$ & $\mathrm{U}$ & $\mathrm{N}$ & 5 \\
\hline Sloane et al. ${ }^{41}$ & $\mathrm{Y}$ & $\mathrm{Y}$ & $\mathrm{U}$ & $\mathrm{Y}$ & $\mathrm{Y}$ & $\mathrm{Y}$ & $\mathrm{Y}$ & $\mathrm{Y}$ & $\mathrm{Y}$ & 8 \\
\hline Thorpe et al. ${ }^{52}$ & $\mathrm{Y}$ & $\mathrm{Y}$ & $\mathrm{U}$ & $\mathrm{N}$ & $\mathrm{Y}$ & $\mathrm{Y}$ & $\mathrm{Y}$ & $\mathrm{Y}$ & $\mathrm{N}$ & 6 \\
\hline van Someren et al. ${ }^{43}$ & $\mathrm{Y}$ & $\mathrm{Y}$ & $\mathrm{U}$ & $\mathrm{N}$ & $\mathrm{Y}$ & $\mathrm{Y}$ & $\mathrm{Y}$ & $\mathrm{U}$ & $\mathrm{N}$ & 5 \\
\hline
\end{tabular}

Note: Q1: Clear cause and effect; Q2: Participants included in comparison similar; Q3: Any comparison other than intervention of interest; Q4: Control group; Q5: Multiple measurements pre and post-intervention; Q6: Follow-up complete; Q7: Outcomes measured in the same way in groups; Q8: Outcomes measured reliably; Q9: Appropriate statistical analysis. Abbreviations: Y: Yes; N: No; U: Unclear; N/A: not applicable.

\section{Light therapy and sleep}

Of the 25 articles assessing the effect of light therapy on sleep disorders, 19 utilized actigraphy. Actigraphy is a reliable technique to study the effect of treatments on sleep and circadian rhythm disorders in people with dementia.60 In addition to using actigraphy, six articles complemented the evaluation of sleep with subjective measures; three of them incorporated an own questionnaire of sleep-wakefulness disturbances, ${ }^{39,46,48}$ and the other three ${ }^{32,33,51}$ included daily sleep reports, plus the Pittsburgh Sleep Quality Index (PSQI)61 in one case ${ }^{32}$ and the Epworth Sleepiness Scale, ESS $^{62}$ in another one. ${ }^{33}$ Other three articles ${ }^{35,37,63}$ only used daily ratings by nursing staff, two articles $^{45,64}$ included only the PSQI, in one study the assessment was made by the Neuropsychiatric Inventory, Nursing Home version (NPI-NH) ${ }^{65}$ and finally, one article used the Sleep Disorders Inventory (SDI) ${ }^{6}$

Only three of the 25 articles reviewed ${ }^{33,45,50}$ found no significant improvement in sleep disturbances. Sekiguchi et al. ${ }^{38}$ found evidence only in 4 out of 17 patients, those with mild to moderate Alzheimer's disease and a shorter duration of illness. 


\section{Light therapy and agitation}

Seven ${ }^{24,30,42,52,53,55,64}$ of the 12 articles evaluating the effect of light therapy on agitation provided significant evidence of potential beneficial effects on this symptom. Three $37,50,63$ found no significant improvement in behavioural symptoms in people with dementia, one ${ }^{51}$ reported only limited evidence of a reduction in agitation, and finally, in the study of Barrick et al. ${ }^{31}$ light therapy resulted in an exacerbation of agitation levels during treatment periods.

\section{Light therapy and mood}

Five out of 10 articles focused on evaluating the use of light therapy for improving mood found a significant decrease in depressive symptoms ${ }^{30,32,53,64,67}$ and another one found more positive emotions, with greater general alertness. ${ }^{50}$ Moreover, Hickman et al. ${ }^{40}$ found limited evidence since depressive symptomatology only decreased in some people with dementia, while other patients showed a worsening of such symptoms. The remaining three articles ${ }^{45,51,63}$ found no benefits of light therapy on mood.

\section{Light therapy and neuropsychiatric symptoms}

Three of the four articles that evaluate the impact of light therapy on neuropsychiatric symptoms (NPS) use the Neuropsychiatric Inventory, two of them ${ }^{49,58}$ use the Nursing Home version (NPI-NH) ${ }^{65}$ and the other one30 utilizes the questionnaire format. ${ }^{68}$ In two of these articles, ${ }^{30,49}$ no significant effects were found neither in the severity of the symptoms either in distress of the caregivers. Conversely, Dowling et al. ${ }^{58}$ found significant ameliorations in depression/dysphoria, aberrant motor behaviour, and appetite/eating disorders, but pointing out the low clinical meaning of the outcomes, with no clinical effect on the agitation/aggression of patients with dementia. The fourth of the articles ${ }^{29}$ on neuropsychiatric symptoms specifically addresses delusions and hallucinations in a small group of patients with Alzheimer's disease. These authors found contradictory results, during the intervention, three of the participants slightly improved the delusional symptoms that they presented before, and another participant did not show delusions neither before nor during the treatment. Conversely, one participant without previous delusional symptomatology developed paranoid delusions and visual hallucinations during the intervention, disappearing one day after the end of the treatment.

\section{Light therapy and cognition}

Only four studies were included in this review. Two of them ${ }^{30,34}$ found significant beneficial effects on cognition decline with increasing Mini-Mental State Examination (MMSE) de Folstein ${ }^{69}$ total scores after light treatment. Nevertheless, other authors ${ }^{48,50}$ found no significant differences in MMSE scores after treatment, nor in the neuropsychological tests developed for the Consortium to Establish a Registry for Alzheimer's Disease (CERAD) ${ }^{70}$ for the measurement of memory, cognitive impairment and the progression of dementia. ${ }^{49}$ 


\section{Light therapy and quality of life}

Münch et al. ${ }^{50}$ reported better quality of life of severely demented patients after light exposure, with better scores using the Quality of Life for Severe Dementia instrument (QUALID) ${ }^{71}$ However, a previous study ${ }^{45}$ reported no significant differences in Quality of Life in Alzheimer's Disease scores (QOL-AD) . ${ }^{72}$

\section{Light therapy and activities of daily living}

Two of the articles ${ }^{30,64}$ included in this review addressed, among others, the effect of light therapy on the functioning of the activities of the participant's daily life,. In one of these studies, ${ }^{64}$ it was used the Minimum Data Set Activities of Daily Living Scale (MDS-ADL) ${ }^{73}$ to measure the dependence in the performance of activities of daily living (ADL), showing a decrease in the scores over time without significant differences. In the other study, ${ }^{30}$ it was found evidence that light therapy attenuated the gradual increase in functional limitations over time in patients with dementia.

\section{Intervention characteristics}

The characteristics of the intervention protocol varied among the studies regarding the type and intensity of the light as well as the duration and frequency of the intervention (see Table 1). In the majority of the articles included in this review, older adults with cognitive impairment were exposed to white bright light, with a high variability of light intensities consisting of doses ranging from $417.24 \operatorname{lux}^{50}$ to 10,000 lux. ${ }^{51,52,53,63,67}$ The most repeated intensity value of the treatment light, appearing in eleven of the 36 selected articles, was a light intensity of 2500 lux, ${ }^{19,24,29,41,44,54}$, $55,56,57,58,59$ followed by the value of 10,000 lux, which was shown in 6 of the articles. On the other hand, four studies used a different type of light treatment. In three ${ }^{32,45,64}$ of them, participants were exposed to a bluish-white light as the treatment condition, and in the other one,${ }^{49}$ it was used as a naturalistic form of light therapy called dawn-dusk simulation (DDS).

With respect to the time of day, excluding McCurry et al. ${ }^{44}$ who provided evening bright light sessions, and Fontana Gasio et al. ${ }^{49}$ who used a special application that includes exposure at dawn and dusk time, all the articles included light intervention in the morning. Ten out of the articles $^{31,40,41,53,54,55,56,57,58,67}$ established experimental designs including both morning and evening bright light, with more significant effects in the morning sessions. Six of the studies $^{30,32,43,45,50,64}$ reported a combined design, with bright light sessions from the time of awakening until 18:00.

Diversity across the studies was also observed in terms of the frequency and duration of light therapy, ranging from $30 \mathrm{~min}^{33,42}$ to $8-10 \mathrm{~h}^{32,64}$ per day, with two hours per day being the most frequent intervention duration, ${ }^{24,29,34,35,36,37,46,47,48,51,54,55,56}$ followed by one hour per day present in seven of the articles. ${ }^{19,38,44,57,58,59,63}$ Two of the studies ${ }^{31,40}$ included an experimental group that was exposed to all-day bright light from 7 a.m. to 8 p.m., but a longer exposure time to the lighting conditions did not demonstrate improved effects. There was also a variety in the light intervention duration, with a minimum of 5 days $^{52}$ and a maximum of $15 \pm 12$ months. ${ }^{30}$ The most common duration of light treatment were periods time of two weeks ${ }^{29,33,37,38,46,47,48,51}$ and four weeks, ${ }^{32,35,36,39,42,43,63,64}$ each period being used respectively in eight of the articles analysed. 
Table 4 includes the most repeated characteristics of light therapy used only in the studies with positive results in any of their outcomes measures.

Table 4. The most repeated characteristics of light therapy in the included studies providing positive results. Intervention characteristics

\begin{tabular}{lllll}
\hline Type of light & Intensity & Time of day & Frequency (time/day) & Total duration \\
White bright light & $2500-10,000 \mathrm{~lx}$ & Morning & $30 \mathrm{~min}-2 \mathrm{~h}$ & $2-4$ weeks \\
\hline
\end{tabular}

\section{Discussion}

This systematic review aimed to analyse studies exploring light therapy in people with cognitive impairment to assess its efficacy as a non-pharmacological approach to manage BPSD, cognition, functional status and quality of life. The current review provides potential evidence that light therapy has positive effects on BPSD, but limited evidence of its effectiveness on cognition, quality of life and functioning in activities of daily living. The secondary objective was to identify the light therapy conditions with the highest benefits for older adults with cognitive impairment, seeking to define guidelines for an adequate protocol in order to facilitate its clinical application with this population. There were great differences between the characteristics of the intervention protocols of the analysed studies, so we synthetized the conditions most commonly used in those studies with positive results.

The articles analysed yielded findings of positive effects of light therapy on BPSD in people with dementia. There is numerous research addressing the effects of light therapy on this symptomatology, especially regarding sleep, agitation and mood.

Most of the publications focus on sleep, reporting significant improvements after or during the intervention, except from four out of the 25 included articles, which found ameliorations only in some of the participants ${ }^{38}$ or no significant changes in sleep disturbances. ${ }^{33,45,50}$ Additionally, better effects on rest-activity rhythms were observed in older people with vascular dementia than those with dementia of Alzheimer's type, probably due to the higher desynchronization of the circadian rhythm in those with vascular dementia. ${ }^{36}$

The second most reported symptom is agitation, with the majority of the publications analysed showing a reduction in it after the intervention with light therapy. Bright light therapy improved agitation symptoms, with a significant decrease in caregiver ratings of physical, verbal and total agitation measured with the Cohen-Mansfield Agitation Inventory (CMAI) ${ }^{74}$ from baseline to the end of the treatment, delaying acrophase of the agitation rhythm; however, it had little effect on observational ratings of agitation. ${ }^{55}$ These authors also concluded that this therapy is more adequate in older people with milder forms of dementia since those with severe dementia possess an SCN that is too degenerated to benefit from light treatment. In contrast to this study, Skjerve et al. ${ }^{39}$ showed improved behavioural symptoms in people with severe dementia, also suggesting a delay in activity rhythms. Other authors ${ }^{30,52,64}$ also used the CMAI for the measurement of agitated behaviours, showing a significant decrease of agitation; in one of these articles, ${ }^{30}$ the positive effects were found only in the condition of light exposure combined with melatonin administration. Additionally, in another study, ${ }^{42}$ bright light therapy showed a positive effect on motor restless behaviour after treatment, with patients being less restless and more cooperative. A potential effect, albeit with limited evidence, was also observed for agitation in severely cognitively impaired patients. ${ }^{51}$ Light therapy is a treatment that may be used at home to reduce agitation. ${ }^{24}$ The effectiveness of light therapy to reduce agitation (with significant effects on four measures of agitation) provides a non-pharmacological alternative to pharmacological treatment, ${ }^{53}$ in which serious side effects are uncommon. ${ }^{39}$ In contrast to this effect of light therapy, other authors $^{31}$ even observed a worsening of behavioural symptoms. Nevertheless, this study includes 
among its limitations that those patients who responded favourably to the light treatment were discharged because of their decreased agitation level. Lyketsos et al. ${ }^{63}$ explain the lack of effect on agitation due to the use of a short-term intervention (4 weeks) or to the absence of a sleep/wake cycle disorder in their study sample. These different outcomes are in conjunction with the different instruments used to measure agitation, the different levels of dementia severity of the patients or the lack of controlling important parameters, as medication consumption or changes before and during the light intervention, among the studies.

Regarding mood, six out of ten articles showed an improvement in depressive symptomatology. In one of these articles, ${ }^{67}$ the effect of light therapy was compared between two groups of participants assigned to them based on their stage of dementia (mild to moderate dementia vs severe dementia). Both groups showed an improvement in total scores, but differences in two subscales of the Depressive Symptom Assessment for Older Adults (DSAOA), ${ }^{75}$ where patients at severe stages of dementia showed greater benefits of the intervention. As opposed to these results, no positive effect of light therapy on mood was found in three articles. ${ }^{45,51,63}$ Moreover, Hickman et al. ${ }^{40}$ found positive effects in some of the participants after the light therapy intervention, but conversely other participants showed a worsening of their depressive symptoms. Circadian disruption increases the likelihood of depressive symptoms, which are also linked to the sleep disturbances ${ }^{76}$ since alterations of rest-activity rhythm contribute to depressive episodes. ${ }^{77}$ Light treatment is also a non-pharmacological option to reduce depression and depressive symptomatology in older adults with dementia, ${ }^{53}$ although the magnitude of the effect may not always be clinically significant. ${ }^{58}$ This effect is large if the efficacy of bright light therapy on depressive symptoms is studied in people with depression or depressive symptomatology at baseline. ${ }^{40}$ These findings are not consistent with those of Burns et al. ${ }^{50}$ who showed no significant differences in Cornell scores, ${ }^{78}$ or those of Lyketsos et al. ${ }^{63}$ who reported no significant changes after bright light exposure in people with dementia. Differences between the methods used to measure depressive symptoms in people with dementia or the collection of information from the caregivers also influence outcomes on the effect of the lighting intervention. ${ }^{40}$

The BPSD with the least research is the neuropsychiatric symptoms, having found only four articles to include in the current review. Three of the articles ${ }^{30,49,50}$ evaluated the impact of light therapy on general NPS, with significant improvements in only one of them. ${ }^{58}$ The fourth article was focus on delusional symptomatology, finding conflicting results among participants of the study: three slightly improved, one showed no change, and another participant began to experience hallucinations and delusions during the intervention. ${ }^{29}$ There is a lack of research regarding the effects of light therapy on psychotic symptoms associated with dementia. Taguchi et al. ${ }^{79}$ examined the use of bright light therapy as a prevention tool of postoperative delirium in a sample of middle-aged and aged hospitalized patients. These authors found that the hallucinations disappeared at the first or second day of the intervention in the experimental group, but they persisted in the control group for several days. Therefore, bright light therapy seems to be useful in the management of NPS, but its application requires caution, as it has been suggested that it may have a significant impact on the onset of delusional symptomology in the course of Alzheimer's disease. $^{29}$

Respecting cognition, we found limited and conflicting evidence for the effects of light therapy. In two of the articles analysed, ${ }^{30,34}$ participants showed an increase in MMSE total scores after the intervention, by contrast in the other two studies, ${ }^{48,50}$ no significant changes were shown in either the MMSE or CERAD scores. Importantly, neuroimaging studies have shown that light regulates cognitive brain activity, which affects cognition and alertness, contributing to sleep and circadian rhythms. ${ }^{80}$ Thus, the potential effect of light on brain functioning must be further explored.

Although the direct effect on the quality of life is not usually considered in the studied articles and is even controversial, improvement of sleep disturbances would also indirectly improve the 
quality of life of people with dementia. ${ }^{76}$ Thus, increased light exposure is associated with significant differences in quality of life in older community-dwelling people. ${ }^{81}$

Likewise, research on the impact of light therapy on functional status in people with cognitive impairment is also scarce, having found for this review only two articles on this topic. The existing studies $^{30,64}$ showed potential benefits of light therapy on the functioning of the participants in their activities of daily living. It is important to promote future research addressing the effects of light therapy on functional status due to its high importance in the field of gerontology and geriatrics.

Finally, regarding the intervention characteristics, it was found high heterogeneity among the protocols of the studies included in the current review. This lack of homogeneity can affect the different outcomes found in the analysed articles. In general, including all the literature addressed, treatment sessions can last from 30 min to $13 \mathrm{~h}$ depending on the equipment used, dosing 250010,000 lux, once or twice a day, for a total intervention period time ranging from 10 days to 10 weeks. With the purpose of providing guidelines for future research in this area, as observed in Table 4, we synthetized the most used characteristics among the studies that resulted in positive effects on the outcome measures. Lastly, the application of light therapy does not require a prescription, but it is important that clinical staff who are familiar with this intervention explain and supervise its application both in institutions and at home. Likewise, although contraindications for light therapy are few, ${ }^{82}$ following up once the intervention has begun is essential to control changes, paying particular attention to the development or increase of neuropsychiatric symptoms, ${ }^{29}$ making adjustments to the intervention protocol if necessary.

\section{Strengths and limitations}

This review should be understood considering some limitations. First, the limited number of people participating in some studies and the heterogeneity of the methodological aspects for light intervention can affect the robustness and clinical relevance of some of the described outcomes and conclusions. In contrast, we carry out an evaluation of the methodological quality for each included study in order to strengthen our results. Additionally, the main strength of this review is the absence of a limit on search years. In addition, we required validated instruments criteria to analyse the effect on BPSD, quality of life and cognitive status in older people, and we presented well-defined aims and explicit inclusion criteria. Besides, we pursued to know the effect of light therapy in older adults (aged 65 years or older) with cognitive impairment, having or no having dementia diagnosis.

\section{Conclusions}

This systematic review showed potential evidence for positive effects of light therapy on managing sleep, behavioural and mood disturbances in people with cognitive impairment, but more research is needed to support these results. A limited effect was found respecting cognition, quality of life and functional status. Despite the great heterogeneity found in the studies, this review reported the most commonly repeated parameters of light therapy used in older adults with cognitive impairment with positive results throughout the literature, thus offering a guide for the design of future studies in this field. Therefore, future studies should be designed to determine the optimal intensity, frequency and duration of the intervention as well to assess its effectiveness.

\section{Declaration of Competing Interest}

None 


\section{Funding}

This work was supported by the Xunta de Galicia (ED431C 2017/49, ED431F 2017/09, FrailNet network IN607C 2016/08, and REGIDEM network IN607C 2017/02). Laura LorenzoLopez was supported by the "Ramon y Cajal” Postdoctoral Senior Grant (RYC-2015-18394) from the Spanish Ministry of Economy and Competitiveness, cofinanced by the European Social Fund. Sponsors had not been involved in the study design, collection, analysis and interpretation of data, in the writing of the report, or in the decision to submit the article for publication.

\section{References}

1. Alzheimer's Disease International. World Alzheimer Report 2018. The state of the art of Dementia research: new frontiers. London, UK: Alzheimer’s Disease International; 2018.

2. Xu J, Qui C. Worldwide Economic Costs and Societal Burden of Dementia. In: Perneczky R, ed. Biomarkers for preclinical Alzheimer's disease, Neuromethods. NewYork, USA: Humana Press; 2018:3-13. https://doi.org/10.1007/978-1-4939-7674-4_1.

3. Azermai M. Dealing with behavioral and psychological symptoms of dementia: a general overview. Psychol Res Behav Manag. 2015;8:181-185. https://doi.org/10.2147/PRBM. S44775.

4. Petrovic M, Hurt C, Collins D, et al. Clustering of Behavioural and Psychological Symptoms in Dementia (BPSD): a European Alzheimer's Disease Consortium (EADC) study. Acta Clin Belg. 2007;62(6):426-432. https://doi.org/10.1179/acb.2007. 062.

5. Cerejeira J, Lagarto L, Mukaetova-Ladinska EB. Behavioral and psychological symptoms of dementia. Front Neurol. 2012;3:1-23. https://doi.org/10.3389/fneur. 2012. 00073.

6. Si Ching L. Managing behavioral and psychological symptoms of dementia- from a geriatrician’s perspective. J Aging Geriatr Psychiatry. 2017;1(3):1-7.

7. Lorenzo-Lopez L, de Labra C, Maseda A, et al. Caregiver's distress related to the patient's neuropsychiatric symptoms as a function of the care-setting. Geriatr Nurs. 2017;38(2):110 118. https://doi.org/10.1016/j. gerinurse. 2016. 08. 004.

8. Cipriani G, Lucetti C, Danti S, Nuti A. Sleep disturbances and dementia. Psychogeriatrics. 2015;15(1):65-74. https://doi.org/10.1111/psyg. 12069.

9. Dyer SM, Harrison SL, Laver K, Whitehead C, Crotty M. An overview of systematic reviews of pharmacological and non-pharmacological interventions for the treatment of behavioral and psychological symptoms of dementia. Int Psychogeriatr. 2018;30(3):295309. https://doi.org/10.1017/S1041610217002344.

10. American Geriatrics Society Beers Criteria Update Expert Panel. American Geriatrics Society 2019 Updated AGS Beers Criteria for Potentially Inappropriate Medication Use in Older Adults. J Am Geriatr Soc. 2019;67(4):674-694. https://doi.org/10.1111/jgs. 15767.

11. Millan-Calenti JC, Lorenzo-Lopez L, Alonso-Bua B, de Labra C, Gonzalez-Abraldes I, Maseda A. Optimal nonpharmacological management of agitation in Alzheimer's disease: challenges and solutions. Clin Interv Aging. 2016;11:175-184. https://doi.org/10.2147/CIA. S69484.

12. Gaviola MA, Inder KJ, Dilworth S, Holliday EG, Higgins I. Impact of individualized music listening intervention on persons with dementia: a systematic review of randomised controlled trials. Australas J Ageing. 2019:1-11. https://doi.org/10.1111/ajag. 12642.

13. Sanchez A, Millan-Calenti JC, Lorenzo-Lopez L, Maseda A. Multisensory stimulation for people with dementia: a review of the literature. Am J Alzheimers Dis Other Demen. 2013;28(1):7-14. https://doi.org/10.1177/1533317512466693.

14. Forrester LT, Maayan N, Orrell M, Spector AE, Buchan LD, Soares-Welser K. Aromatherapy

for dementia. Cochrane Database Syst Rev. 2014;2: CD003150. https://doi.org/10.1002/14651858. CD003150. pub2.

15. Woods B, O'Philbin L, Farell EM, Spector AE, Orrell M. Reminiscence therapy for dementia. Cochrane Database Syst Rev. 2018;3: CD001120. https://doi.org/10.1002/14651858. CD001120. pub3. 
16. Hanford N, Figueiro M. Light therapy and Alzheimer's disease and related dementia: past, present, and future. J Alzheimers Dis. 2013;33(4):913-922. https://doi.org/10.3233/JAD2012-121645.

17. Mitolo M, Tonon C, La Morgia C, Testa C, Carelli V, Lodi R. Effects of light treatment on sleep, cognition, mood, and behavior in Alzheimer's disease: a systematic review. Dement Geriatr Cogn Disord. 2018;46:371-384. https://doi.org/10.1159/000494921.

18. Forbes D, Blake CM, Thiessen EJ, Peacock S, Hawranik P. Light therapy for improving cognition, activities of daily living, sleep, challenging behaviour, and psychiatric disturbances in dementia. Cochrane Database Syst Rev. 2014;2: CD003946. https://doi.org/10.1002/14651858. CD003946. pub4.

19. Dowling GA, Hubbard EM, Mastick J, Luxenberg JS, Burr RL, Van Someren EJW. Effect of morning bright light treatment for restactivity disruption in institutionalized patients with severe Alzheimer's disease. Int Psychogeriatr. 2005;17(2):221-236. https://doi.org/10.1017/S1041610205001584.

20. van Maanen A, Meijer AM, van der Hejiden KB, Oort FJ. The effects of light therapy on sleep problems: a systematic review and meta-analysis. Sleep Med Rev. 2016;29:52-62. https://doi.org/10.1016/j. smrv. 2015. 08. 009.

21. Sloane PD, Figueiro M, Cohen L. Light as therapy for sleep disorders and depression in older adults. Clin Geriatr. 2008;16(3):25-31. 22. Chong MS, Tan KT, Tay L, Wong YM, Ancoli-Israel S. Bright light therapy as part of a multicomponent management program improves sleep and functional outcomes in delirious older hospitalized adults. Clin Interv Aging. 2013;8:565-572. https://doi.org/10.2147/CIA. S44926.

23. Abraha I, Rimland JM, Trotta FM, et al. Systematic review of systematic reviews of nonpharmacological interventions to treat behavioural disturbances in older patients with dementia. The SENATOR-OnTop series. BMJ Open. 2017;7:(3) e012759. https://doi.org/10.1136/bmjopen-2016-012759.

24. Lovell BB, Ancoli-Israel S, Gevirtz R. Effect of bright light treatment on agitated behavior in institutionalized elderly subjects. Psychiatry Res. 1995;57(1):7-12. https://doi.org/10.1016/0165-1781(95)02550-G.

25. Forbes D, Morgan DG, Bangma J, Peacock S, Adamson J. Light therapy for managing sleep, behaviour, and mood disturbances in dementia. Cochrane Database Syst Rev. 2004;2: CD003946. https://doi.org/10.1002/14651858. cd003946. pub2.

26. Terman M. Evolving applications of light therapy. Sleep Med Rev. 2007;11:497-507. https://doi.org/10.1016/j. smrv. 2007. 06. 003.

27. Moher D, Liberati A, Tetzlaff J, Altman DG. The PRISMA Group. Preferred reporting items for systematic reviews and meta-analyses: the PRISMA statement. PLoS Med. 2009;6:(7) e1000097. https://doi.org/10.1371/journal. pmed. 1000097.

28. Tufanar C, Munn Z, Aromataris E, Campbell J, Hopp L. Chapter 3: systematic reviews of effectiveness. In: Aromataris E, Munn Z, eds. Joanna Briggs Institute Reviewers’ Manual.

The Joanna Briggs Institute 2017.

https://wiki.joannabriggs.org/display/MANUAL/Chapter+3\%3A+Systematic+reviews+of+e ffectiveness. 2017 (Accessed 15 November 2019).

29. Schindler SD, Graf A, Fischer P, T€olk A, Kasper S. Paranoid delusions and hallucinations and bright light therapy in Alzheimer's disease. Int J Geriatr Psychiatry. 2002;17:10711072. https://doi.org/10.1002/gps. 497.

30. Riemersma-van der Lek RF, Swaab DF, Twisk J, Hol EM, Hoogendijk WJG, Van Someren EJW. Effect of bright light and melatonin on cognitive and noncognitive function in elderly residents of group care facilities. JAMA. 2008;299(22):2642-2655. https://doi.org/10.1001/jama. 299. 22. 2642.

31. Barrick AL, Sloane PD, Williams CS, et al. Impact of ambient bright light on agitation in dementia. Int J Geriatr Psychiatry. 2010;25(10):1013-1021. https://doi.org/10.1002/gps. 2453.

32. Figueiro MG, Hunter CM, Higgins P, et al. Tailored lighting intervention for persons with dementia and caregivers living at home. Sleep Health. 2015;1(4):322-330. https://doi.org/10.1016/j. sleh. 2015. 09. 003.

33. Friedman L, Spira AP, Hernandez B, et al. Brief morning light treatment for sleep/wake disturbances in older memory-impaired individuals and their caregivers. Sleep Med. 2012;13(5):546-549. https://doi.org/10.1016/j. sleep. 2011. 11. 013.

34. Graf A, Wallner C, Schubert V, et al. The effects of light therapy on mini-mental state examination scores in demented patients. Biol Psychiatry. 2001;50(9):725-727. https://doi.org/10.1016/S0006-3223(01)01178-7. 
35. Mishima K, Okawa M, Hishikawa Y, Hozumi S, Hori H, Takahashi K. Morning bright light therapy for sleep and behavior disorders in elderly patients with dementia. Acta Psychiatr Scand. 1994;89(1):1-7. https://doi.org/10.1111/j. 1600-0447. 1994.tb01477. x.

36. Mishima K, Hishikawa Y, Okawa M. Randomized, DIM light controlled, crossover test of morning bright light therapy for rest-activity rhythm disorders in patients with vascular dementia and dementia of Alzheimer's type. Chronobiol Int. 1998;15(6):647-654. https://doi.org/10.3109/07420529808993200.

37. Satlin A, Volicer L, Ross V, Herz L, Campbell S. Bright light treatment of behavioral and sleep disturbances in patients with Alzheimer's disease. Am J Psychiatry. 1992;149(8):1028-1032. https://doi.org/10.1176/ajp. 149. 8. 1028.

38. Sekiguchi H, Iritani S, Fujita K. Bright light therapy for sleep disturbance in dementia is most effective for mild to moderate Alzheimer's type dementia: a case series. Psychogeriatrics. 2017;17(5):275-281. https://doi.org/10.1111/psyg. 12233.

39. Skjerve A, Holsten F, Aarsland D, Bjorvatn B, Nygaard HA, Johansen IM. Improvement in behavioral symptoms and advance of activity acrophase after short-term bright light treatment in severe dementia. Psychiatry Clin Neurosci. 2004;58(4):343-347. https://doi.org/10.1111/j. 1440-1819. 2004. 01265. x.

40. Hickman SE, Barrick AL, Williams CS, et al. The effect of ambient bright light therapy on depressive symptoms in persons with dementia. J Am Geriatr Soc. 2007;55 (11):18171824. https://doi.org/10.1111/j. 1532-5415. 2007. 01428. x.

41. Sloane PD, Williams CS, Mitchell M, et al. High-intensity environmental light in dementia: effect on sleep and activity. J Am Geriatr Soc. 2007;55(10):1524-1533. https://doi.org/10.1111/j. 1532-5415. 2007. 01358. x.

42. Haffmans PMJ, Sival RC, Lucius SAP, Cats Q, Van Gelder L. Bright light therapy and melatonin in motor restless behaviour in dementia: a placebo-controlled study. Int J Geriatr Psychiatry.2001;16(1):106-110.

https://doi.org/10.1002/1099-1166(200101)16:1<106::AIDGPS288>3. 0. CO;2-9.

43. Van Someren EJW, Kessler A, Mirmiran M, Swaab DF. Indirect bright light improves circadian rest-activity rhythm disturbances in demented patients. Biol Psychiatry. 1997;41(9):955-963. https://doi.org/10.1016/S0006-3223(97)89928-3.

44. McCurry SM, Pike KC, Vitiello MV, Logsdon RG, Larson EB, Teri L. Increasing walking and bright light exposure to improve sleep in community-dwelling persons with Alzheimer's disease: results of a randomized, controlled trial. J Am Geriatr Soc. 2011;59(8):1393-1402. https://doi.org/10.1111/j. 1532-5415. 2011. 03519. x.

45. Sloane PD, Figueiro M, Garg S, et al. Effect of home-based light treatment on persons with dementia and their caregivers. Light Res Technol. 2015;47(2):161-176. https://doi.org/10.1177/1477153513517255.

46. Fetveit A, Skjerve A, Bjorvatn B. Bright light treatment improves sleep in institutionalized elderlyan open trial. Int J Geriatr Psychiatry. 2003;18(6):520-526. https://doi.org/10.1002/gps. 852.

47. Fetveit A, Bjorvatn B. The effects of bright-light therapy on actigraphical measured sleep last for several weeks post-treatment. A study in a nursing home population. J Sleep Res. 2004;13(2):153-158. https://doi.org/10.1111/j. 1365-2869. 2004. 00396. x.

48. Fetveit A, Bjorvatn B. Bright-light treatment reduces actigraphic-measured daytime sleep in nursing home patients with dementia: a pilot study. Am J Geriatr Psychiatry. 2005;13(5):420-423. https://doi.org/10.1097/00019442-200505000-00012.

49. Fontana Gasio P, Kr€auchi K, Cajochen C, et al. Dawn-dusk simulation light therapy of disturbed circadian rest-activity cycles in demented elderly. Exp Gerontol. 2003;38(12):207-216. https://doi.org/10.1016/S0531-5565(02)00164-X.

50. M€unch M, Schmieder M, Bieler K, et al. Bright light delights: effects of daily light exposure on emotions, rest activity cycles, sleep and melatonin secretion in severely demented patients. Curr Alzheimer Res. 2017;4(10):1063-1075. https://doi.org/10.2174/1567205014666170523092858.

51. Burns A, Allen H, Tomenson B, Duignan D, Byrne J. Bright light therapy for agitation in dementia: a randomized controlled trial. Int Psychogeriatr. 2009;21(4):711-721. https://doi.org/10.1017/S1041610209008886.

52. Thorpe L, Middleton J, Russell G, Stewart N. Bright light therapy for demented nursing home patients with behavioral disturbance. Am J Alzheimers Dis. 2000;15(1):18-26. https://doi.org/10.1177/153331750001500109.

53. Onega LL, Pierce TW, Epperly L. Effect of bright light exposure on depression and agitation in older adults with dementia. Issues Ment Health Nurs. 2016;37(9):660-667. https://doi.org/10.1080/01612840. 2016. 1183736. 
54. AncoliIsrael S, Martin JL, Kripke DF, Marler M, Klauber MR. Effect of light treatment on sleep and circadian rhythms in demented nursing home patients. J Am Geriatr Soc. 2002;50(2):282-289. https://doi.org/10.1046/j. 1532-5415. 2002. 50060. x.

55. Ancoli-Israel S, Martin JL, Gehrman P, et al. Effect of light on agitation in institutionalized patients with severe Alzheimer disease. Am J Geriatr Psychiatry. 2003;11(2):194-203. https://doi.org/10.1097/00019442-200303000-00010.

56. Ancoli-Israel S, Gehrman P, Martin JL, et al. Increased light exposure consolidates sleep and strengthens circadian rhythms in severe Alzheimer's disease patients. Behav Sleep Med. 2003;1(1):22-36. https://doi.org/10.1207/S15402010BSM0101_4.

57. Dowling GA, Mastick J, Hubbard EM, Luxenberg JS, Burr RL. Effect of timed bright light treatment for rest-activity disruption in institutionalized patients with Alzheimer's disease. Int J Geriatr Psychiatry. 2005;20(8):738-743. https://doi.org/10.1002/gps. 1352.

58. Dowling GA, Graf CL, Hubbard EM, Luxenberg JS. Light treatment for neuropsychiatric behaviors in Alzheimer's disease. West J Nurs Res. 2007;29(8):961-975. https://doi.org/10.1177/0193945907303083.

59. Dowling GA, Burr RL, Van Someren EJW, et al.Melatonin and bright-light treatment for rest-activity disruption in institutionalized patients with Alzheimer's disease. J AmGeriatr Soc. 2008;56(2):239-246. https://doi.org/10.1111/j. 1532-5415. 2007. 01543. x.

60. Ancoli-Israel S, Cole R, Alessi C, Chambers M, Moorcroft W, Pollak CP. The role of actigraphy in the study of sleep and circadian rhythms. Sleep. 2003;26(3):342-392. https://doi.org/10.1093/sleep/26. 3. 342.

61. Buysse DJ, Reynolds CF, Monk TH, Berman SR, Kupfer DJ. The Pittsburgh sleep quality index: a new instrument for psychiatric practice and research. Psychiatry Res. 1989;28(2):193-13. https://doi.org/10.1016/0165-1781(89)90047-4.

62. Johns M. A new method for measuring daytime sleepiness: the Epworth sleepiness scale. Sleep. 1991;14(6):540-545. https://doi.org/10.1093/sleep/14. 6. 540.

63. Lyketsos CG, Veiel LL, Baker A, Steele C. A randomized, controlled trial of bright light therapy for agitated behaviors in dementia patients residing in long-term care. Int J Geriatr Psychiatry. 1999;14(7):520-525. https://doi.org/10.1002/(SICI)1099-1166(199907)14:7<520::AID-GPS983>3. 0. CO;2-M.

64. Figueiro MG, Plitnick BA, Lok A, et al. Tailored lighting intervention improves measures of sleep, depression, and agitation in persons with Alzheimer's disease and related dementia living in long-term care facilities. Clin Interv Aging. 2014;9:1527-1537. https://doi.org/10.2147/CIA. S68557.

65. Wood S, Cummings JL, Hsu M, et al. The use of the Neuropsychiatric Inventory in nursing home residents. Characterization and measurement. Am J Geriatr Psychiatry. 2000;8(1):7583. https://doi.org/10.1097/00019442-200002000-00010.

66. Tractenberg RE, Singer CM, Cummings JL, Thal LJ. The Sleep Disorders Inventory: an instrument for studies of sleep disturbance in personswith Alzheimer's disease. J Sleep Res. 2003;12(4):331-337. https://doi.org/10.1046/j. 0962-1105. 2003. 00374. x.

67. Onega LL, Pierce TW, Epperly L. Bright light therapy to treat depression in individuals with mild/moderate or severe dementia. Issues Ment Health Nurs. 2018;39(5):370-373. https://doi.org/10.1080/01612840. 2018. 1437648.

68. Kaufer DI, Cummings JL, Ketchel P, et al. Validation of the NPI-Q, a brief clinical form of the Neuropsychiatric Inventory. J Neuropsychiatry Clin Neurosci. 2000;12(2):233-239. https://doi.org/10.1176/appi. neuropsych. 12. 2. 233.

69. Folstein MF, Folstein SE, McHugh PR. Mini-Mental State. A practical state method for grading the cognitive state of patients for the clinician. J Psychiatric Res. 1975;12:189-198. https://doi.org/10.1016/0022-3956(75)90026-6.

70. Morris JC, Heyman A, Mohs RC, et al. The Consortium to Establish a Registry for Alzheimer's Disease (CERAD). Part I. Clinical and neuropsychological assessment of Alzheimer's disease. Neurology. 1989;39(9):1159-1165. https://doi.org/10.1212/WNL. 39. 9. 1159.

71. Weiner MF, Martin-Cook K, Svetlik DA, Saine K, Foster B, Fontaine CS. The quality of life in late-stage dementia (QUALID) scale. J Am Med Dir Assoc. 2000;1(3):114-116.

72. Logsdon RG, Gibbons LE, McCurry SM, Teri L. Quality of life in Alzheimer's disease: patient and caregiver reports. J Mental Health Aging. 1999;5(1):21-32.

73. Hartmaier SL, Sloane PD, Guess HA, Koch GG. The MDS Cognition Scale: a valid instrument for identifying and staging nursing home residents with dementia using the Minimum Data Set. J Am Geriatr Soc. 1994;42(11):1173-1179. https://doi.org/10.1111/j. 1532-5415. 1994. tb06984. x. 
74. Cohen-Mansfield J. Marx M. S. Rosenthal A. S. A description of agitation in a nursing home. J Gerontol19898;44(3):7784. 10. 1093/geronj/44. 3. M77.

75. Onega LL. Content validation of the depressive symptom assessment for older adults. Issues Ment Health Nurs. 2008;29(8):873-894. https://doi.org/10.1080/01612840802182920.

76. Figueiro MG. Light, sleep and circadian rhythms in older adults with Alzheimer's disease and related dementias. Neurodegener Dis Manag. 2017;7(2):119-145. https://doi.org/10.2217/nmt-2016-0060.

77. Smagula SF, Krafty RT, Thayer JF, Buysse DJ, Hall MH. Rest-activity rhythm profiles associated with manic-hypomanic and depressive symptoms. J Psychiatr Res. 2018;102:238-244. https://doi.org/10.1016/j. jpsychires. 2018. 04. 015.

78. Alexopoulos GS, Abrams RC, Young RC, Shamoian CA. Cornell scale for depression in dementia. Biol Psychiatry. 1988;23(3):271-284. https://doi.org/10.1016/00063223(88)90038-8.

79. Taguchi T, Yano M, Kido Y. Influence of bright light therapy on postoperative patients: a pilot study. Intens Crit Care Nurs. 2007;23(5):289-297. https://doi.org/10.1016/j. iccn. 2007. 04. 004.

80. Gaggioni G, Maquet P, Schmidt C, Dijk DJ, Vandewalle G. Neuroimaging, cognition, light and circadian rhythms. Front Syst Neurosci. 2014;8:1-12. https://doi.org/10.3389/fnsys. 2014. 00126.

81. Kume Y, Makabe S, Singha-Dong N, Vajamun P, Apikomonkon H, Griffiths J. Seasonal effects on the sleepwake cycle, the restactivity rhythm and quality of life for Japanese and Thai older people. Chronobiol Int. 2017;34(10):1377-1387. https://doi.org/10.1080/07420528. 2017. 1372468.

82. Lack L. Wright H. The use of bright light in the treatment of insomnia. In: Perlis M, Aloia M, Kuhn B, eds. Behavioral Treatment for Sleep Disorders: a Comprehensive Primer of Behavioural Sleep Medicine Interventions. London: Academic Press; 2011:e1e6. 10. 1016/B978-0-12-381522-4. 00042-0. 